\title{
Function of Flotillins in Receptor Tyrosine Kinase Signaling and Endocytosis: Role of Tyrosine Phosphorylation and Oligomerization
}

\author{
Nina Kurrle, Bincy John, Melanie Meister and Ritva Tikkanen
}

Additional information is available at the end of the chapter

http://dx.doi.org/10.5772/48598

\section{Introduction}

There are two homologous members of the flotillin family which have been designated as flotillin-1/reggie-2 and flotillin-2/reggie-1, owing to their almost simultaneous discovery by two separate research groups $(1,2)$. In 1997, these proteins were described under the name "reggies", since they were found to be upregulated during the regeneration of retinal ganglion cells of the goldfish, following an optic nerve lesion (2). In the same year, Bickel et al. independently discovered them as components of detergent resistant fractions, and due to their ability to float in density gradients ascribed them as flotillins (1).

Biological membranes are complex structures composed of numerous proteins and lipids. As shown in Figure 1A, flotillin-1 and flotillin-2 are both constitutively associated with sphingolipid and cholesterol enriched membrane microdomains, known as lipid rafts (1, 35). Especially flotillin-1 is often considered as a bona fide marker protein for lipid rafts. The lipid raft concept was first postulated in 1997 by Kai Simons and Elina Ikonen (6), and has since then been refined and reshaped throughout the years. Nowadays, lipid rafts can be described as fluctuating nanoscale membrane assemblies composed of proteins, sphingolipids and cholesterol, which can coalesce to form platforms that are important for cell signaling, viral infection and membrane trafficking (7). It has been proposed that proteins with a high affinity for an ordered lipid environment, often achieved by posttranslational modifications, will preferentially associate with lipid rafts (8). We have shown that flotillin proteins are linked to the plasma membrane by means of fatty acid modifications, especially myristoylation and palmitoylation (4), and both modifications facilitate the interaction with membrane cholesterol (our unpublished results). Earlier studies proposed that flotillins are localized in caveolae-type of lipid rafts, but later findings 
demonstrated that flotillins rather reside outside of caveolae and are thought to define their own subtype of lipid rafts (9).

The first description of flotillin-2 actually took place in 1994, when the group of Madeleine Duvic cloned an N-terminally truncated version of flotillin-2 from human epidermal keratinocytes and named it epidermal surface antigen (ESA) (10). However, in later studies by the same group, it turned out that the true ESA (ECS-1 antigen) is actually distinct from flotillin-2 (11). The flotillin-2 cDNA originally cloned by Schroeder et al. represents a truncated protein with a lower molecular mass that has not been detected in vivo and most likely came about due to a missing 5' end of the cloned cDNA and false assignment of the ATG start codon. A monoclonal antibody ECS-1 raised against human keratinocytes was used for the identification of ESA. Curiously, a very similar approach with a monoclonal antibody against cell surface proteins was used by the Stuermer group to identify reggie1/flotillin-2 and reggie-2/flotillin-1 as regeneration proteins of goldfish retinal ganglion cells (2). In this case, the true antigen of the M802 antibody was later shown to be identical with Thy-1, a glycophosphatidyl-inositol (GPI)-linked neuronal protein (12). Since flotillins have been shown not to traverse the membrane, it is unlikely that they were directly recognized by the antibodies used for the screenings. A more likely explanation is that flotillins were associated with the true antigens as molecular complexes and thus accidentally discovered. This is also supported by the data showing that flotillins are generally strongly associated with GPI-linked proteins (13-15). However, the details of their interaction have mechanistically not been clarified, since neither flotillins nor GPI-anchored proteins completely traverse the membrane. Thus, their interaction may be mediated by a yet to be identified transmembrane protein. Accordingly, we were able to show that both flotillins are coprecipitated after immunoprecipitation of surface biotinylated membrane proteins in confluent human epithelial MCF10A cells (our unpublished results).

Both flotillin-1 and flotillin-2 show a constitutive expression in almost all mammalian cell types and are highly conserved from fly to man (reviewed in 16). Furthermore, flotillin-like proteins are also present in bacteria (17), plants (18), fungi and metazoans but are absent in yeast and Caenorhabditis elegans (19). In humans, a $15 \mathrm{~kb}$ single-copy flotillin-1 gene with 13 exons is located on chromosome 6 (6p21.3), giving rise to a $1.9 \mathrm{~kb}$ mRNA transcript. The ORF encodes a 427 residue protein with a molecular mass of $47 \mathrm{kDa}$ (20). A single copy of flotillin-2 gene is located on chromosome 17 (17q11-12) which encodes for a $48 \mathrm{kDa}$ protein (10). Human flotillin-1 shares $98 \%$ identity with the murine protein and $47 \%$ identity with human flotillin-2. Flotillins are classified as part of the SPFH (Stomatin/ Prohibitin/ Flotillin/HflK/C) superfamily of proteins, also called prohibitin homology (PHB) proteins $(21,22)$. Different from other members of the SPFH superfamily, flotillins exhibit a conserved domain termed the flotillin domain C-terminally to the SPFH domain (Fig. 1B). This domain is characterized by several repeats of glutamic acid and alanine (EA repeats) and is predicted to form coiled coil structures $(23,24)$. A few years ago, the crystal structure of the core SPFH domain of mouse flotillin-2 was solved, and it shows partial similarity to the respective segment of Pyrococcus horikoshii stomatin (25). This structural region includes amino acids 43 to 173 of the SPFH domain of flotillin-2 and depicts that flotillin-2 SPFH 
domain has a compact, ellipsoid-globular structure, comprising four alpha helices and six beta strands. Although the structure of the flotillin domain has not yet been solved, it is predicted to show a high propensity for alpha helices. This could explain why flotillin domains are engaged in coiled coil structures that are important for the oligomerization of flotillins.
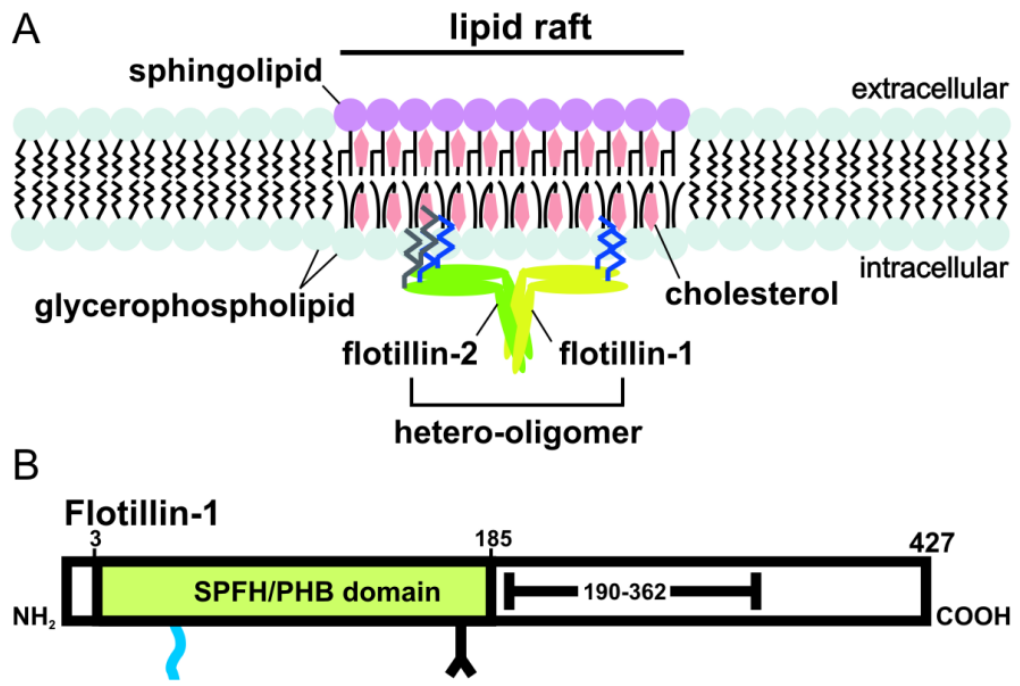

Flotillin-2

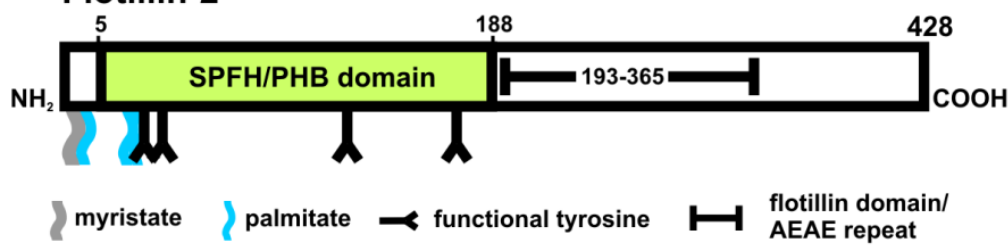

Figure 1. Flotillin-1 and flotillin-2 form oligomers in lipid rafts. A: Flotillin-1 and flotillin-2 are associated with specialized membrane microdomains, known as lipid rafts. Lipid rafts are defined as fluctuating nanoscale assemblies in membranes, composed of certain proteins and enriched in sphingolipids and cholesterol. They can coalesce to form platforms that are important for cell signaling, viral infection and membrane trafficking. Flotillin- 1 and flotillin- 2 are linked to the membrane by acylation, namely palmitoylation (both flotillins) and myristoylation (only flotillin-2). An important feature of flotillin function is the formation of homo- and hetero-oligomers. B: Flotillins contain in their $\mathrm{N}$-terminal region a domain homologous with the prohibitin family of proteins, whereas their Cterminus is characterized by the unique flotillin domain with AEAE repeats.

\section{Modifications, oligomerization and trafficking of flotillins}

Flotillin-1 and flotillin-2 are ubiquitously expressed proteins and exhibit a relatively complementary tissue distribution. Northern blot analysis showed a high tissue-specific expression of flotillin-1 in murine brain, fat and heart tissue (1). High protein levels of 
flotillin-1 and flotillin-2 were found, e.g. in murine lung, thymus, bladder, cerebrum and hypothalamus tissue lysates (24, our unpublished results). Additionally, it has been shown that the expression of flotillin-2 increases during differentiation and myogenesis of $\mathrm{C} 2 \mathrm{C} 12$ cells, whereas flotillin-1 increases during 3T3-L1 cell adipogenesis (1) and osteoclastogenesis (26). In contrast, during differentiation of rat pheochromocytoma PC12 cells, no change in the expression of flotillin-1 or flotillin-2 was observed (24).

The subcellular localization of flotillin- 1 and flotillin-2 is as diverse as their tissue expression pattern and seems to be cell type specific. In glandular cervical cancer HeLa cells, PC12 cells, human hepatocellular carcinoma HepG2 cells, Chinese hamster ovary $(\mathrm{CHO})$ cells and human breast cancer MCF-7 cells, flotillins mainly reside at the inner leaflet of the plasma membrane and to a minor extent in vesicular structures. A major localization in vesicular structures is found in astrocytes, the human HaCaT keratinocyte cell line and mouse embryonic fibroblast (MEF) cells. Colabeling experiments and immunogold electron microscopy studies identified the vesicular structures as endosomes and lysosomes in astrocytes, Jurkat, PC12 and HeLa cells (13, 14, 27-30). In line with the previous results, endogenous labeling of flotillin-2 in MCF10A cells shows a more prominent localization at the plasma membrane, whereas flotillin- 1 is found in vesicular structures in non-confluent cells. However, with increasing confluency and advanced differentiation, flotillin-1 translocates to the plasma membrane (our unpublished results), a feature already observed in differentiating mouse 3T3-L1 cells $(31)$. Rajendran et al. $(32,33)$ showed that flotillin-1 and flotillin-2 reside in preassembled structures under resting conditions in immature and mature hematopoietic cells, but they can move into uropods during cell migration and in immunological synapses during T-cell activation. These two studies highlight the fact that the subcellular localization of flotillin proteins is highly versatile and depends on the cell type and the differentiation status.

Total internal reflection fluorescence (TIRF) microscopy revealed that flotillin-1 and flotillin2 exhibit a vesicular cycling at the plasma membrane (28), which seems to be independent of clathrin and caveolin $(9,13,34,35)$. Microtubule disruption in HeLa cells results in the accumulation of flotillin-1/flotillin-2 positive vesicles in the cytosol (28), while the same treatment in murine neuroblastoma N2a cells did not affect flotillin-2 localization (36). Manipulation of the actin cytoskeleton might also have a cell type dependent influence on the mobility of flotillins. In FRAP (fluorescence recovery after photobleaching) experiments, an increased lateral mobility of flotillin-2 after disruption of the actin cytoskeleton with cytochalasin D in N2a cells was observed, while treatment with the actin stabilizing compound jasplakinolide reduced the lateral mobility. On the contrary, the use of cytochalasin D in HeLa cells had no significant effect on flotillin-2 mobility (36). Recently, Affentrager et al. (37) showed that a dynamic actin turnover is required for capping of flotillin-1 and flotillin-2 in primary human peripheral blood T-lymphocytes, which underscores the importance of the actin cytoskeleton for flotillin localization and trafficking. The lateral motility is also dependent on the localization itself, since at least in Jurkat T cells, flotillin-2 is selectively immobilized in the region of the preformed cap and only shows a high lateral mobility at the plasma membrane outside the cap (29). In other studies, flotillin- 
1 was found in exosomes of rat and human reticulocytes, human erythroleukemia K562 cells, lymphoid-B Daudi cells, human breast carcinoma MCF-7 cells and in exosomes of the cerebrospinal fluid (38-40). Flotillin-2 was detected in exosomes derived from MCF-7 cells and oligodendroglial Oli-neu cells $(40,41)$. Flotillin-1 was additionally found in mature phagosomes of murine J774 macrophages (42) and in the nucleus of human prostate cancer PC-3 cells (43).

Localization and trafficking of flotillins in the Golgi apparatus is still controversial. Nevertheless, evidence suggests that the involvement of Golgi in flotillin trafficking is also a cell type-specific process. In non-differentiated PC12, normal rat kidney (NRK), CHO and HeLa cells, flotillin-1 localizes to the Golgi complex (44-46). Also in rat brain tissue sections, flotillin-1 was found in the Golgi (47). In contrast, Morrow et al. (3) reported that flotillin-1 reaches the plasma membrane via a brefeldin A-resistant pathway in baby hamster kidney (BHK) cells. However, they did not rule out the possibility of Golgi involvement in flotillin-1 trafficking, as a GFP-tagged construct of the flotillin-1 SPFH domain colocalized with the cis-medial Golgi marker GM130 during early times of expression. Less is known about flotillin-2 and Golgi complex association. Only the study of Langhorst et al. (28) showed by immunogold EM microscopy that flotillin-2 was localized in vesicles in the Golgi field in Jurkat cells, but not in Golgi stacks.

Chemokines, growth factors and certain proteins can affect the localization and the trafficking of flotillin proteins. In Satb2-negative neuronal cells, flotillin-1 becomes more clustered in response to semaphorin 3a treatment, which acts as a potent guidance cue presented to cortical axons in vivo (48). In primary human peripheral blood T-lymphocytes, flotillin-1 and flotillin-2 are randomly distributed along the inner leaflet of the plasma membrane, and upon stimulation with stromal cell derived factor 1 (SDF-1), flotillins form stable caps in the uropod $(32,37)$. The best studied growth factor that has an effect on flotillin trafficking is the epidermal growth factor (EGF), as originally shown by us (30). Recently, we showed that flotillin-1 is indeed involved in the activation and downstream signaling of the EGF receptor (EGFR) (27). Upon stimulation with EGF, flotillin-2 becomes tyrosine phosphorylated at several tyrosine residues by Src kinases (30). This phosphorylation event is dependent on the activation of the EGFR, since the EGFR autophosphorylation inhibitor AG1478 prevents flotillin-2 phosphorylation. In regard to its localization, EGF stimulation results in a Tyr163-dependent translocation of flotillin-2 into the late endosomal compartment (30). Experiments in the group of B. Nichols confirmed our findings stating that Y163 is important for flotillin-2 internalization. In addition, it was shown that the corresponding tyrosine in flotillin-1, Y160, is crucial for flotillin-1 internalization (49). Other growth factors, such as hepatocyte growth factor (HGF) or insulin also affect the localization of flotillin proteins (Figure 2, and our unpublished results). Figure 2 shows that ten minutes after HGF stimulation, flotillin- 1 is translocated from the plasma membrane to late endosomes/lysosomes where it colocalizes with LAMP3.

Posttranslational modifications of eukaryotic proteins are often important cues for localization, trafficking and membrane association of proteins (50). Two important modifications are $\mathrm{N}$-myristoylation and S-palmitoylation. N-myristoylation is an irreversible 
unstimulated

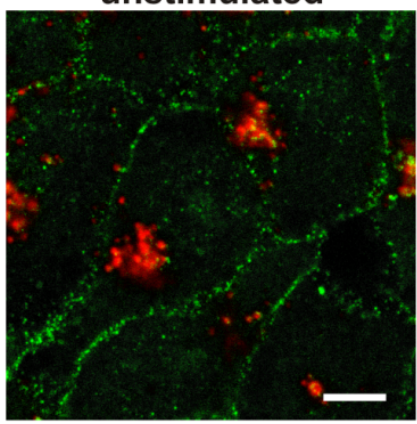

$10 \mathrm{~min}$ HGF

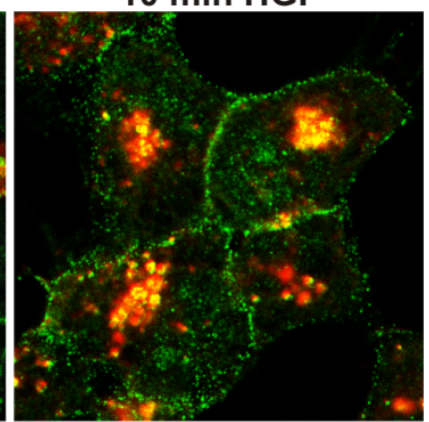

Figure 2. Flotillin-1 translocates to late endosomes upon $10 \mathrm{~min}$ of hepatocyte growth factor (HGF) stimulation. Staining of endogenous flotillin-1 (green) and endogenous lysosome-associated membrane glycoprotein 3 (LAMP-3; red) in HeLa cells stimulated for 10 minutes with HGF. Scale bar: $15 \mu \mathrm{m}$

acylation reaction at an N-terminal glycine residue, which is catalyzed by the enzyme Nmyristoyl transferase (51). S-palmitoylation is a reversible reaction, in which the acyl group is transferred to cysteine residues by palmitoyl acyltransferases, referred to as the DHHC family $(52,53)$. In some proteins, e.g. Src-related protein tyrosine kinases, which are both myristoylated and palmitoylated, the myristoylation reaction is a prerequisite for the palmitoylation to occur (54). Our experiments with overexpressed flotillin-2 constructs in HeLa cells showed that flotillin-2 is myristoylated at Gly2 and palmitoylated at Cys4, Cys19 and Cys20, and Cys4 seems to be the major palmitoylation site (4). The myristoylation at Gly2 is essential for membrane targeting of flotillin-2, as the mutant flotillin-2 Gly2Ala remains fully soluble upon overexpression. Furthermore, the mutation of the cysteine residues to alanine and the concomitant loss of palmitoylation sites resulted in more soluble flotillin-2 mutants (4). In the study by Li et al. (53), the palmitoyl acyltransferase DHHC5 was identified as the enzyme to palmitoylate flotillin-2. In neuronal stem cells of DHHC5 gene-trapped mice, flotillin-2 palmitoylation was abolished. For flotillin-1, which is not myristoylated, it was shown that plasma membrane localization is dependent on palmitoylation of a conserved cysteine residue (Cys34) within its SPFH domain (3). This finding is supported by the study of Affentrager et al. (37), in which overexpressed flotillin-1 Cys34Ala exhibited a diffuse cytosolic localization in human T-lymphoblasts. In contrast, palmitoylation of this cysteine seems not to play a crucial role for plasma membrane targeting in adipocytes. Instead, deletion of the second hydrophobic stretch (residues 134 151) results in a similar cytoplasmic localization (31). This finding suggests that the effect of Cys34 palmitoylation in flotillin-1 function might be cell type specific.

Oligomeric proteins are prevalent in nature and comprise approximately one third of all known proteins. From the perspective of protein evolution, oligomerization may be of advantage for a functional control such as allosteric regulation (55). In the case of flotillin proteins, oligomerization is an essential feature for membrane raft association, endocytosis and EGF signaling $(30,56)$. Flotillin- 1 and flotillin-2 form both homo- and hetero-oligomers $(4,5,23,34)$, and Solis et al. (23) suggested that flotillin oligomers exist in a monomer- 
tetramer equilibrium. The homo-oligomerization of flotillin-2 is mediated by the C-terminal half of the protein (amino acid residues 208-428), which seems to enhance the association of flotillin-2 with the plasma membrane and its membrane raft localization $(4,29)$. The Cterminal half of flotillin-1 and flotillin-2 contains the so called flotillin-domain, which contains several EAEA repeats and is predicted to form three adjacent coiled-coil structures. The formation of flotillin oligomers was shown to be dependent on the second and partially on the first coiled-coil structure (23). Using the flotillin-2 Y163A mutant, we showed that not the phosphorylation as such but hetero-oligomerization with flotillin-1 is indispensable for flotillin-2 endocytosis. In addition, our experimental data implicate that after EGF stimulation, preexisting flotillin oligomers form larger oligomeric complexes in which the stoichiometry of 1:1, also shown by Frick et al. (34), is preserved (56). In summary, these data show that not only the coiled coil structures within the C-terminal half of flotillins are important for the oligomerization, but also the tyrosine residue 163 in flotillin-2 plays a role in the hetero-oligomerization with flotillin-1.

Results of the Stuermer group have shown that ectopic expression of the presumed oligomerization domain of flotillin-2 comprising the amino acids 184 to 390 in Jurkat T cells interferes with function of flotillins in these cells and prevents the formation of macrodomains upon crosslinking of GM1 by cholera toxin (29). Hence, this fragment, termed R1EA, most likely prevents the proper oligomerisation of flotillins, thus functioning in a trans-negative manner. Upon overexpression of R1EA, insulin-like growth factor induced neurite outgrowth was abrogated in N2a neuroblastoma cells, and axon differentiation was impaired in hippocampal neurons (57). In addition, in R1EA expressing cells, recruitment of CAP/ponsin, which interacts with flotillin-1, to focal contacts was affected, resulting in imbalanced activation of Rho family GTPases Rac1 and Cdc42. Interestingly, focal adhesion kinase (FAK) activity was increased by R1EA expression. However, other signaling pathways such as ERK1/2, PKC, and PKB signals were unaltered (57). In line with this, previous studies have shown that upon growth factor stimulation of PC12 cells, protein kinase Pyk2, ubiquitin ligase Cbl and the CAP homolog ArgBP-2 are recruited to lipid rafts (58). Moreover, cholesterol depletion resulted in a diminished neurite outgrowth, suggesting involvement of membrane rafts (58). It has been proposed that ArgBP-2 associates with $\mathrm{Cbl}$ and Pyk2 via its $\mathrm{SH} 3$ domain and recruits them to lipid rafts by interacting with flotillin-1 through its SoHo domain (58). These findings point towards a vital role of flotillins in cytoskeletal remodeling and neurite growth at least in cultured cells. However, neither the flotillin-1 knockout mouse model (59) nor flotillin-2 deficient mice (our unpublished data) show a major neuronal phenotype. It thus remains to be clarified if flotillins are only required under conditions of neuronal regeneration or if they are generally important for the physiology of neurons in mammals.

\section{In search of the molecular function of flotillins}

As can be expected from such highly conserved and ubiquitous proteins, flotillins have been shown to play a role in a large number of vital cellular processes. Their original discovery as "reggies" suggested a function in neuronal regeneration in the goldfish $(2,45)$, which has 
been later verified by further studies from the same group (57, 60, 61). However, the regenerative capacity of the optic nerve is poorly conserved in mammals, making a generalization of the regenerative function of flotillins in the nervous system of mammals difficult. Recent studies have confirmed the relevance of flotillins for axon regeneration in zebrafish as well as in mammalian cell cultures. Apparently, downregulation of flotillins by flotillin specific morpholinos in the zebrafish resulted in a massive reduction in the number of regenerating axons (60). Since the role of flotillins in neuronal regeneration has recently been directly reviewed, we here omit a long discussion of the topic. Instead, the reader is referred to the recent review of C. Stuermer (62).

Flotillins also appear to be involved in membrane trafficking processes such as endocytosis and phagocytosis $(13,34,42)$, and they have been suggested to be involved in the regulation the actin cytoskeleton $(4,11,57)$. One emerging important function of flotillins, especially of flotillin-1, is the regulation of membrane receptor signaling, e.g. through mitogen activated protein kinases (MAPK) $(27,30,43,56,63-67)$. Below, we have summarized the data on the function of flotillins in some of these processes.

\section{Flotillins in endocytosis}

During their discovery, flotillins were described to be associated with membrane rafts (1, 24). First findings suggested that flotillins would be integral membrane proteins of the cavelike invaginations called caveolae $(1,24)$, but these findings have later been disputed by several other studies $(3,9,13,34)$. However, the raft association of flotillins appears to be important for their function, and flotillin-1 has even been suggested to define a novel nonclathrin, non-caveolin endocytosis pathway that would operate via membrane rafts $(13,34)$. In eukaryotic cells, uptake of membrane bound molecules such as receptors can take place by means of several different pathways. The best characterized and the most common pathway for endocytosis is mediated by clathrin coated vesicles which are formed at the plasma membrane by the assembly of a coated pit which then forms a clathrin coated vesicle. However, recent research from several groups has revealed that endocytosis can be divided into numerous pathways, many of which do not require clathrin, and are thus designated as clathrin-independent. For a general introduction on endocytosis, the reader is referred to these excellent reviews (68-70). Some factors that regulate the clathrin independent pathways have been identified in the recent years, and it is clear that small GTPases such as Cdc42, Rho A or Arf (ADP ribosylation factor) family are involved, each determining their own endocytic pathway. The role of flotillins in endocytosis has recently been reviewed in detail (71), and we thus here only present a brief overview and a summary of the very recent studies not included in the review of Otto and Nichols.

Early after their discovery, flotillins were shown to associate and colocalize with GPIanchored proteins. A role in the internalization of GPI-anchored proteins such as CD59 and of the glycosphingolipid GM1 has been demonstrated $(13,34,72)$. Flotillins have also been suggested to be involved in the endocytosis of the GPI-linked Nodal coreceptor cripto (73). Although flotillins are affirmed regulators of cholera toxin subunit B endocytosis $(13,72)$, which is mediated by the binding of the toxin to GM1, no evidence for a direct role of 
flotillins in the endocytosis of the Shiga toxin or the plant toxin ricin was found (46). However, flotillins were shown to control other trafficking steps of these toxins, most likely a retrograde transport step towards Golgi/ER. Intriguingly, depletion of flotillins resulted in a higher degree of mannosylation of ricin, and the toxicity of both ricin and Shiga toxin was increased (46). Thus, flotillins are important factors in controlling not only the endocytosis but also other steps of the cellular trafficking of various molecules.

In addition to GPI-anchored proteins, flotillins have recently been shown to participate in the protein kinase $C$ (PKC) induced endocytosis of neurotransmitter receptors such as the dopamine transporter DAT (also designed as solute carrier family 6, member 3 or SLC6A3) and glial glutamate transporter, GLT1 (74). PKC was shown to phosphorylate flotillin-1 at a Ser residue, and this phosphorylation was required for the endocytosis of DAT (74). Recent publications have revealed a role for flotillins in the endocytosis of the Alzheimer amyloid precursor protein (APP), the amyloidogenic processing of which requires its endocytosis (75). In addition, Niemann-Pick C1-like 1 (NPC1L1), an important mediator of the uptake of dietary cholesterol, relies on flotillins for its endocytosis, and depletion of flotillins drastically reduces cholesterol uptake in cultured cells (76). Curiously, APP, NPC1L1 and DAT are all endocytosed by means of clathrin mediated endocytosis, whereas flotillins as raft associated proteins have been rather suggested to define a non-clathrin endocytosis pathway, and there is very little colocalization between clathrin and flotillins (13). Thus, it is possible that rather than directly mediating raft dependent endocytosis through flotillin microdomains, flotillins might exert their function by first facilitating the uptake of some cargo molecules into rafts and their efficient clustering, but the act of endocytosis may only take place after transfer of the cargo into clathrin coated pits. Hence, it will be important in the future to study the exact relationship of flotillins and clathrin dependent endocytosis.

We have recently shown that flotillins are involved in EGFR/MAP kinase signaling, and siRNA mediated downregulation of flotillin-1 results in severe impairment of both EGFR phosphorylation and MAPK signaling (27). Since flotillins also become phosphorylated upon EGFR signaling (30), it would not be surprising if they were involved in EGFR endocytosis, which has been shown to take place by means of both clathrin dependent and raft mediated endocytosis $(77,78)$. However, according to our recent findings, flotillin-1 depletion does not significantly affect the EGF-induced endocytosis of EGFR (27). Furthermore, EGFR is efficiently endocytosed even in cells depleted of both clathrin and flotillin-1 (our unpublished data). Interestingly, recent findings from the Stuermer group have implicated that depletion of flotillin-2/reggie-1 may affect the endocytosis of EGFR (79). This finding is somewhat counter intuitive as flotillin-2 depletion induces a significant depletion of flotillin-1 as well, and flotillin-2 knockdown cells express only minor amounts of both flotillins. Flotillin-1 knockdown cells in turn still exhibit substantial flotillin-2 expression. Thus, these results imply that the flotillin-2 homo-oligomers that are still present in flotillin-1 knockdown cells may be capable of supporting normal EGFR endocytosis, whereas in the absence of flotillins, EGFR uptake from the plasma membrane is impaired. However, one also has to keep in mind some important methodological differences between our study (27) and that of Solis et al. (79). Our EGF uptake assays were performed with 
surface-bound iodinated EGF using short endocytosis times (up to $6 \mathrm{~min}$ ), which allows the calculation of endocytosis rate constants for the receptor, providing a reliable and truly quantitative measurement of the early endocytosis and uptake of the receptor from the plasma membrane (80). We observed no difference between flotillin-1 knockdown and control cells even after very short endocytosis times (2, 4 or $6 \mathrm{~min})$. However, Solis et al. used methods such as continuous uptake of fluorescent EGF for 5-10 min, or endocytosis of biotinylated EGFR for $120 \mathrm{~min}$. In the latter assay, they observed an increased amount of EGFR remaining at the cell surface of flotillin-2 knockdown cells. However, also the total EGFR was increased in flotillin-2 depleted cells after 120 min EGF stimulation. Since substantial degradation of EGFR (and EGF) will evidently take place over such a long time, these results might indicate that the degradation and not endocytosis of EGFR is impaired, resulting in generally higher amount of remaining EGFR in flotillin-2 knockdown cells. Furthermore, when longer time points are used (10 - $120 \mathrm{~min})$, some of the receptor will also be recycled back to the plasma membrane, thus biasing these results. In fact, considerable recycling of EGFR has been shown to take place in A431 cells (81). Hence, an increased amount of EGFR on the surface of flotillin-2 knockdown cells could also be a result of increased recycling combined with a reduced degradation. In addition to this, the A431 cells used by Solis et al. massively overexpress EGFR and are thus widely considered as a poor model system for EGFR trafficking, since the high amounts of EGFR may result in saturation of the endocytosis pathways normally used by the receptor and redirection of the receptor to pathways that otherwise play a negligible role. Therefore, the role of each flotillin in EGFR endocytosis should be reevaluated and directly compared in the same cell system with methods that are suitable for unbiased measurement of endocytosis, such as iodinated EGF uptake.

One important open question on the role of flotillins in endocytosis is if the flotillinmediated endocytosis of various cargo molecules is dependent on the GTPase dynamin or not, since the studies have resulted in contradictory results, depending on the cell system used and cargo molecules studied. Since flotillins are endocytosed from the plasma membrane as a result of EGF stimulation (30) but appear not to directly affect EGFR endocytosis (27), they may in some cases also themselves represent cargo molecules whose cellular localization needs to be regulated during signaling. It is possible that depending on the endocytic process, cargo and stimulus, mechanistically different pathways are used. For a further discussion of this idea, please refer to the recent review of Otto and Nichols (71). Another important question is how the modifications (such as phosphorylation) and oligomerization of flotillins affect the trafficking of their putative cargo molecules. This topic is discussed more in detail below in the chapter "Functional significance of phosphorylation and oligomerization of flotillins".

\section{Functions of flotillins in cell migration and adhesion}

Already the discovery of flotillins by using antibodies directed against cell surface proteins led to the assumption of a functional role of flotillin proteins in cell adhesion $(2,82)$. Up to date, much has been speculated about this attribute, but only a few studies actually showed 
an involvement of flotillins in the establishment and maintenance of cell-cell and cell-matrix adhesion structures. One of the first functional hints was obtained in the study by Hoehne et al. (83), in which they investigated the functional role of flotillins in the model organism Drosophila melanogaster. The null mutant of flotillin-2 in Drosophila is viable and showed no visible phenotype, whereas Mz1369-Gal4 regulated overexpression of flotillin-2 alone or together with flotillin-1 resulted in a severe disturbance of the ommatidial pattern in regard to the number of primary pigment cells and cone cells. Furthermore, staining with antibodies against the cell adhesion molecules Roughest, Kin-of-irre and Sticks-and-Stones of the IgCAM protein family showed a strong labeling of an abnormal accumulation of multivesicular bodies in interommatidial cells. These observations led to the conclusion that flotillins interfere with the distribution of cell adhesion and signaling molecules in imaginal discs of Drosophila. In 2009, Málaga-Trillo et al. (15) published that the loss of the prion protein $(\mathrm{PrP})$ in zebrafish embryos is characterized by the loss of embryonic cell adhesion. They presented confocal images of drosophila S2 cells, in which overexpressed mouse PrPEGFP colocalized with overexpressed rat flotillin-2-dsRed at cell-cell contact sites. Similar results were obtained with overexpressed zebrafish PrP-2-EGFP with rat flotillin-1-dsRed and flotillin-2-dsRed as well as zebrafish flotillin-1a-dsRed. (15)

One major type of cell-cell contact in multicellular organisms is the adherens junction. The core of adherens junctions is composed of proteins of the cadherin and catenin family (84). Insights into a functional association of flotillins with this cell-cell contact type were obtained in the study of Bodrikov et al. (85), who showed that a transient knockdown of flotillin-2 in primary hippocampal neurons perturbed axon growth and differentiation. This phenotype was rescued in $62 \%$ of the neurons by overexpression of the constitutive active mutant of the GTPase TC10 which participates in the exocyst dependent polarized delivery of cargo to the growth cone (86). Interestingly, the amount of coprecipitated of N-Cadherin, which is a core protein of adherens junctions in adult neuronal cells, increased in flotillin-2 immunoprecipitates from mouse hippocampal HPL3-4 cells after application of PrP-Fc. Overexpression of a dominant negative TC10 blocked this effect, which indicates that the PrP together with flotillin-2 is important for the recruitment of N-Cadherin, which in turn takes place in a TC10-dependent manner (85). Very recently, the Stuermer group could show that flotillins together with the PrP play a role in E-Cadherin recruitment to adherens junctions (79). They showed that both proteins colocalized with E-cadherin at the plasma membrane in human epidermoid carcinoma A431 cells. Knockdown of flotillin-2 or PrP resulted in an abnormal shape of adherens junctions, causing overlapping of neighboring cells (79). In addition, electron microscopy revealed that the length of adherens junctions was shorter in flotillin-2 and PrP knockdown cells (79).

The complexing of the junctional proteins p120-catenin (p120ctn) and N-Cadherin at cell-cell contact sites was shown to occur in cholesterol rich membrane domains in mouse C2C12 cells (87). A similar observation was made in differentiated human colon adenocarcinoma HT-29 cells, in which the interaction of E-Cadherin with p120ctn preferentially takes place in lipid rafts. A stable knockdown of flotillin-1 resulted in a diffuse localization of p120ctn and an impaired recruitment of p120ctn and E-Cadherin in lipid rafts. In addition, flotillin-1 
depletion decreased the enzymatic activity of ALP and DPPIV during the enterocytic differentiation process (88). This study revealed a novel function of flotillin-1 in a lipid raft mediated maturation of adherens junctions and in intestinal cell differentiation.

Other studies implicated that flotillins seem to be of functional relevance for cell-matrix adhesion processes. We showed that the transient knockdown of flotillin-2 in HeLa cells impaired cell spreading on fibronectin, as compared to cells transfected with control siRNA (30). Overexpression of flotillin-2-EGFP enhanced cell spreading and induced filopodia-like protrusions in an expression-level dependent manner in several epithelial cells lines, e.g. $\mathrm{HaCaT}$ cells $(4,30)$. The observed enrichment of flotillin- 2 fusion proteins at cell-cell contact sites and the induction of filopodia suggest an active link to the cytoskeleton. Indeed, use of in vivo colocalization assays, in vitro binding assays and actin polymerization assays suggested that flotillin-2 interacts with F-actin via its SPFH domain, and that this binding mediates the anchoring of the actin cytoskeleton to the plasma membrane (36).

\section{Role of flotillins in EGF receptor and mitogen activated protein kinase signaling}

In the last 12 years, various studies have shown that flotillin- 1 and flotillin- 2 are physically and functionally linked to signal transduction pathways of several membrane receptors, especially receptor tyrosine kinases such as the insulin receptor (IR), the nerve growth factor (NGF) receptor, tropomyosin-related kinase A (TrkA), the polymeric Immunoglobulin E (IgE) receptor, fibroblast growth factor receptor (FGFR) and the epidermal growth factor receptor (EGFR) $(27,30,63,65,89,90)$. However, the mechanisms how flotillins may regulate the signaling process have not been dissected in detail. Only recently, we have shed light on the molecular mechanisms of flotillin-1 function in EGFR/MAPK signaling $(27,89)$. We showed that flotillin-1 actually plays a dual role by first regulating the clustering of EGFR in the plasma membrane after EGF stimulation and, later on during signaling, by functioning as a MAPK scaffolder protein (27). We also demonstrated that flotillin-1, but not flotillin-2, interacts with Fibroblast Growth Factor Receptor Substrates 2 and 3 (FRS2 and FRS3) (89) which have previously been shown to be involved in MAPK signaling (91-93). (See Figure 3).

Depletion of flotillin-1 in HeLa cells results in a decreased tyrosine phosphorylation of the EGFR especially at Tyr1173 already after a short EGF stimulation, and the reduced phosphorylation persists up to a time point where no phosphorylation of EGFR can be detected (27). By means of TIRF microscopy, we showed that the formation of EGFR clusters at the cell surface upon EGF stimulation is impaired after flotillin-1 knockdown. This fits well with the findings of Schneider et al. (75) who showed that flotillins might be important for the clustering of APP on the cell surface before its endocytosis. In addition to EGFR clustering, flotillin-1 depletion also prevents the association of EGFR and some of its downstream signaling partners with membrane rafts (27). Although the fraction of total cellular EGFR that is found in rafts is very low, this might be the population of the receptors that is capable of fast signaling upon stimulation. Earlier findings from Hofman et al. have suggested that the raft associated EGFR actually resides in two separate populations of rafts which then coalesce after stimulation, bringing the required signaling molecules in close 


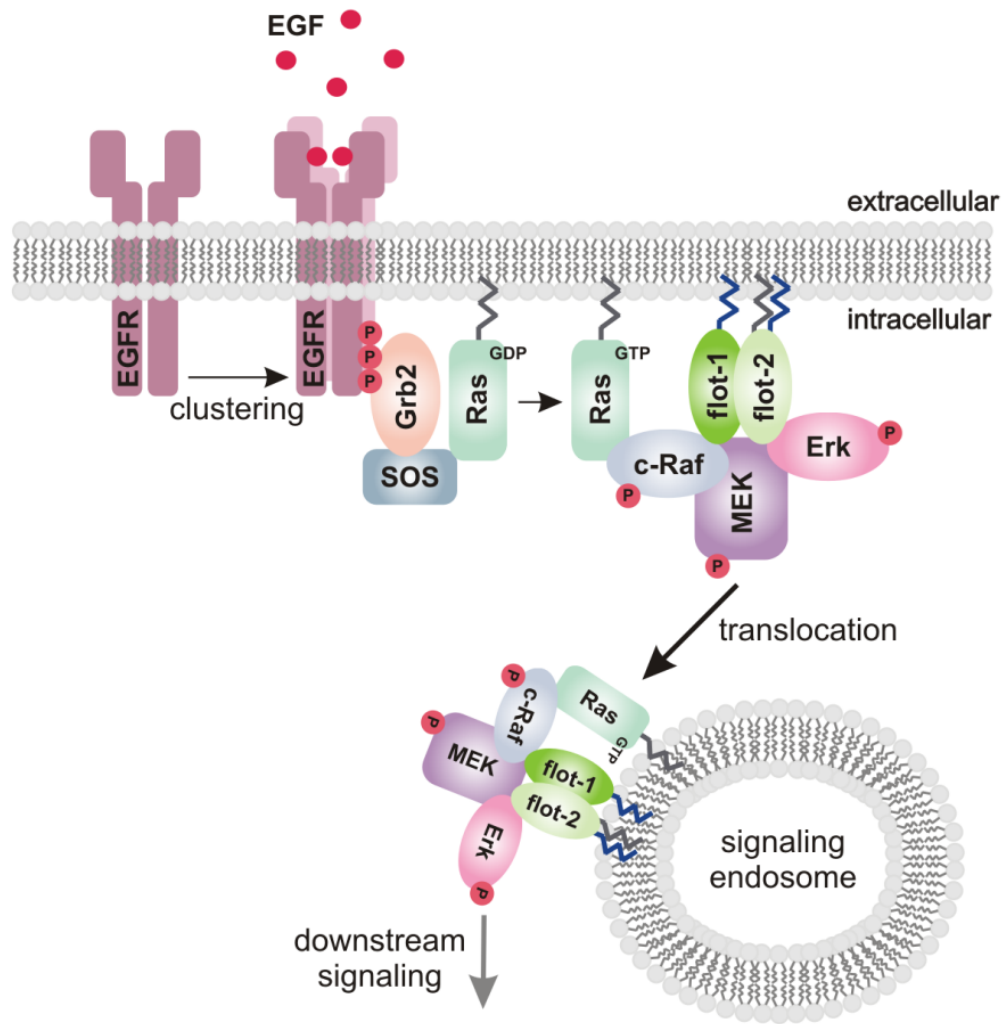

Figure 3. Flotillin-1 is a MAPK scaffolder - the role of flotillins in EGFR/MAPK signaling. Binding of the ligand EGF results in dimerization and activation of the EGFR. Thereby, several Tyr residues in its kinase domain become either autophosphorylated or phosphorylated by other kinases. These phosphorylation events lead to the recruitment of further signaling components, especially those of the MAPK signaling pathway. Grb2 binds to the phosphorylated EGFR kinase domain and transmits the downstream signal via Ras, c-Raf and MEK to the MAPK ERK1/2. The EGF-induced signaling through MAPK is initiated at the plasma membrane but later on translocates to specialized endosomes, i.e. signaling endosomes. Flotillins are able to associate with the EGFR itself and regulate the clustering of the receptor at the plasma membrane. Flotillin-1 directly interacts with the components of the MAPK signaling pathway, e.g. c-Raf, MEK, KSR1 and ERK and can thus be classified as a novel MAPK scaffolder.

contact (94). This model is well in line with our earlier findings showing that upon EGF stimulation, the molecular mass of flotillin oligomers increases, most likely due to their coalescence into larger complexes (56).

We also observed a reduced downstream signaling towards the canonical MAPK pathway and Protein Kinase B/Akt after stimulation with EGF or basic fibroblast growth factor (bFGF) upon flotillin-1 knockdown (27), implicating that flotillin-1 is not only involved in EGFR but also in FGFR signaling. Similar results with EGF have later on been obtained by 
Solis et al. (79) in A431 cells, which overexpress EGFR to a very high degree, after flotillin-2 depletion. This is somewhat in contrast to our unpublished results in HeLa cells, which express physiological amounts of EGFR. In these cells, depletion of flotillin-2, resulting in nonexpression of flotillin-1, actually causes an increased MAPK phosphorylation upon EGF stimulation (A. Banning \& R.T., unpublished findings). This again stresses the importance of using analogous cell systems for studies in signaling, as the degree of downstream signaling is highly dependent on the amount of EGFR expressed.

Flotillin-1 depletion also resulted in reduced activation of the MAPK pathway, especially of ERK kinases, which are required for transmitting the MAPK signal into the nucleus (27). Although flotillin-1 depletion clearly impairs an early step, EGFR clustering, during the signaling, which alone would be sufficient to inhibit MAPK signaling, this appears not to be the only reason for the reduced ERK activity. Incubation of the cells with phorbol myristate acetate (PMA) results in the activation of PKC, which in turn can active the cRAF kinase in the absence of an upstream EGFR signal $(95,96)$. Our attempt to overcome the downstream ERK inhibition by PKC-mediated activation of cRAF resulted in normal activation of cRAF and MEK kinases, whereas the ERK phosphorylation could not be rescued in flotillin-1 knockdown cells (27). This demonstrates that flotillin-1 must play a role not only at the plasma membrane but also at the level of ERK activation. Accordingly, we showed that flotillin-1 directly interacts with several MAPK pathway proteins, namely cRAF, MEK1 and ERK2. This interaction was not dependent on the MAPK scaffolder Kinase Suppressor of Ras (KSR), since flotillin-1 was found to coprecipitate with the MAPK component also in KSR1 depleted cells (27). Thus, these results suggest that flotillin-1 is indeed a bona fide MAPK scaffolder that is capable of directly interacting with several MAPK pathway proteins. Interestingly, the flotillin-1 interactor FRS2 has also been suggested to function as a scaffolding protein for the MAPK proteins $(92,97)$. Furthermore, our yeast two-hybrid studies have revealed another MAPK scaffolding protein, Mitogen-activated Protein Kinase Organizer 1 (MORG1) as an interaction partner of flotillins (our unpublished data). In future studies, it will be interesting to dissect the details of the regulation of MAPK signaling by flotillin-1, e.g. how these complexes are regulated during signaling in terms of their molecular composition and cellular localization.

The role of flotillins in EGFR signaling is supported by our recent findings in stable flotillin1 and flotillin-2 knockdown human breast cancer MCF-7 cells (Figure 4). Comparable to HeLa cells, in MCF7 cells the stability of flotillin-1 is strongly dependent on flotillin-2, since the knockdown of flotillin-2 resulted also in a loss of flotillin-1. However, knockdown of flotillin-1 had no effect on flotillin-2 expression. Immunoblot analysis showed that the amount of EGFR is increased in flotillin-1 and flotillin-2 knockdown cells (Figure 4A). This effect is only visible in stable knockdown cells and not in transient knockdown cells, most likely due to an adaption of the EGFR amount to overcome the decreased EGFR-MAPK signaling in flotillin knockdown cells. We have previously observed this effect also in HeLa cells and other cell lines (our unpublished results). The EGFR, also known as ErbB1/Her1, is one of the four known members of the ErbB/Her protein-tyrosine kinase family (reviewed in (98)). Analysis of the protein amount of ErbB2/Her2 and ErbB3/Her3 showed no detectable 
A

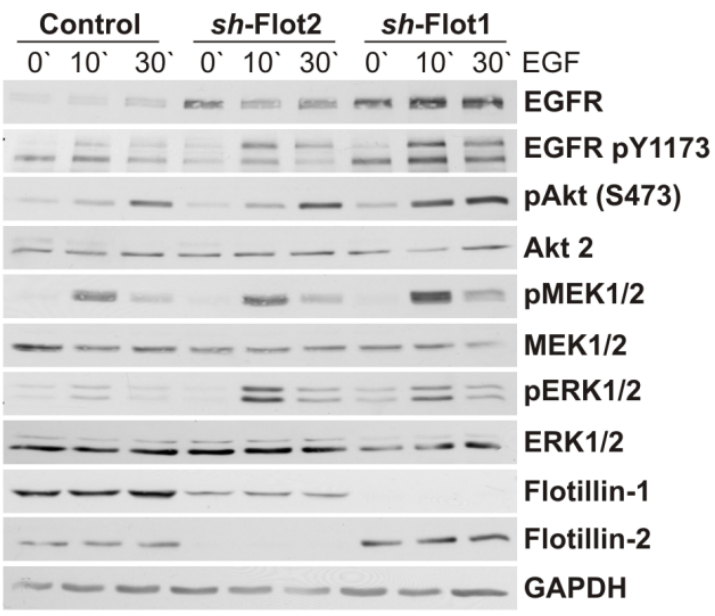

B

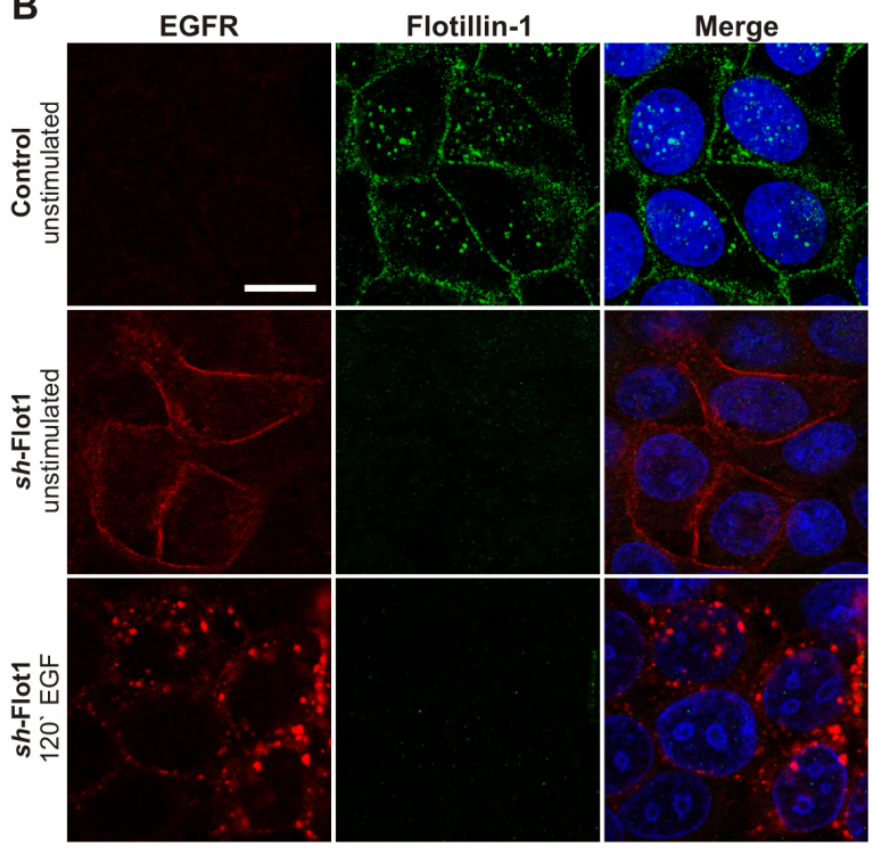

Figure 4. The reduced activation of the MAPK signaling pathway due to fiotillin depletion is compensated by an increased protein expression level of the epidermal growth factor receptor (EGFR) in MCF-7 cells. (A) Stimulation of stable flotillin-1 and flotillin-2 MCF-7 knockdown cells with EGF for 10 minutes and 30 minutes. The reduced activation of the MAPK signaling in flotillin knockdown cells is compensated by an upregulation of the EGFR protein level. (B) lmmunofluorescence of endogenous EGFR (red) and endogenous flotillin-1 (green) in unstimulated and EGF-stimulated (120 min) flotillin-1 knockdown cells (sh-Flot1). Control cells are shown only in unstimulated state, due to undetectable EGFR level by immunofluorescence. Scale bar: $20 \mu \mathrm{m}$ 
change in the protein level (data not shown), which indicates that flotillin depletion specifically affects the protein level of the EGFR. In general, the endogenous EGFR is not detectable by immunofluorescence in MCF-7 cells, whereas in flotillin-1 depleted MCF-7 cells, a prominent staining at the plasma membrane is visible (Figure 4B). Stimulation with EGF for 120 minutes results in a translocation of the EGFR in vesicular structures in these cells. This is in line with our previous results in HeLa cells, indicating that the endocytosis of the EGFR is not affected by flotillin-1 depletion (27).

\section{Flotillins and other receptor tyrosine kinases}

The first experimental evidence for a functional role of flotillins in insulin signaling was presented by the Saltiel group (63). They demonstrated that flotillin-1 forms a ternary complex with c-Cbl-associated protein (CAP) and the E3 ubiquitin protein ligase Cbl upon insulin stimulation (Figure 5). This was shown to be important for lipid raft association, downstream signaling of the CAP-Cbl complex and finally for glucose uptake by the glucose transporter GLUT4 in 3T3-L1 adipocytes (63). Previously, the interaction of CAP with $\mathrm{Cbl}$ was shown to be independent of insulin stimulation in vivo and in vitro and to be mediated by SH3 domains $(99,100)$. In line with this, expression of CAP $\triangle \mathrm{SH} 3$ inhibited the translocation of GLUT4 from storage vesicle to the plasma membrane upon insulin stimulation (63). Further analysis of the flotillin-1 interaction mode with murine CAP isoforms showed that flotillin-1 only interacts with the isoform CAP-4 via its first hydrophobic domain, but not with the isoforms CAP-1, -2, and -3 (31). In CAP, the sorbin homology domain (SoHo) seems to be important for flotillin-1 binding, as the expression of CAP $\triangle$ SoHo prevented the translocation of Cbl to lipid rafts in 3T3-L1 adipocytes (101). Since the murine CAP isoforms 1-4 all contain the SoHo domain, a predicted 70 amino acids long proline-rich region $\mathrm{N}$-terminal to the SoHo domain in CAP-4 might also be of importance for flotillin-1 binding (102). While flotillin-1 was found in perinuclear regions in differentiated skeletal muscle cells (103), it was located at the plasma membrane in adipocytes (31) under basal conditions, suggesting that the role of flotillin-1 in targeting GLUT4 to the plasma membrane after insulin stimulation may differ between muscle and adipose tissue. Indeed, Fecchi et al. (103) showed that flotillin-1 colocalized with GLUT4 in the perinuclear region of myotubes, and both proteins translocated to the sarcolemma in a PI3K/ PKC $\zeta$ - and caveolin-3 dependent manner upon stimulation with insulin. In addition, it was demonstrated that caveolin-3 is involved in the PI3K-dependent insulin signaling pathway, whereas flotillin-1 is crucial for the PI3K-independent insulin signaling pathway by interacting with the main scaffolding protein CAP. The interaction with CAP was also shown for flotillin-1 and flotillin-2 in mouse neuroblastoma N2a cells, in which overexpressed flotillin-1 and flotillin-2 colocalized with endogenous CAP at focal adhesion sites (57). This result indicates that by means of their interaction with CAP, flotillins might play an additional role in focal adhesion dynamics and integrin signaling.

Receptor tyrosine kinases of the Trk receptor family regulate various cellular processes, such as proliferation and differentiation, through several cellular signal cascades. Three types of Trk receptors exist in humans, including TrkA, TrkB and TrkC, which have varying 
affinities to neutrotrophins such as NGF. TrkA is specifically activated by NGF (reviewed in $(104,105)$. Downstream signaling by TrkA seems to be dependent on the lipid raft association of TrkA. Upon NGF stimulation, TrkA is recruited to lipid rafts in PC12 cells, a process dependent on its association with CAP, which in turn binds to flotillin-1 (65). The deletion of the CAP SoHo domain abolishes the lipid raft association of TrkA and further downstream signaling events such as ERK1/2 phosphorylation (65). (See Figure 5)
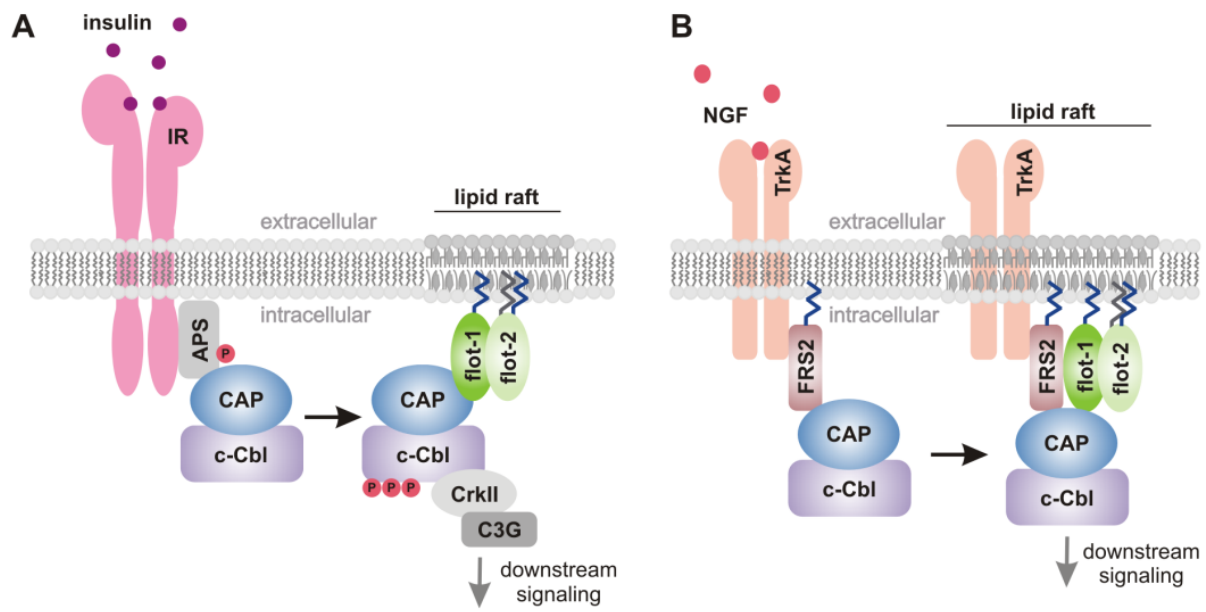

Figure 5. Proposed functional role of flotillin proteins in insulin and NGF signaling cascades. (A) Insulin is the major hormone controlling essential metabolic processes such as glucose and lipid turnover. Insulin binding to the extracellular subunits of the insulin receptor tyrosine kinase (IR) induces a conformational change and results in the autophosphorylation of several tyrosine residues in the cytoplasmic part of the IR. In adipocytes, the constitutive complex of the E3 ubiquitin ligase c-Cbl with the $\mathrm{c}-\mathrm{Cbl}$ associated protein (CAP) is recruited to the active receptor. The interaction of $\mathrm{CAP} / \mathrm{c}-\mathrm{Cbl}$ complex with the IR was found to be indirect and mediated via the Adapter protein with Pleckstrin homology and Src homology 2 domains (APS). To initiate downstream signaling events, the CAP/c-Cbl complex then dissociates from the IR and is recruited to lipid rafts by forming a ternary complex with flotillin-1. (B) Receptor tyrosine kinases of the Trk receptor family regulate various cellular processes in the mammalian neuronal system, such as proliferation and differentiation by activation through neutrophins. The receptor TrkA is activated by the neutrophin nerve growth factor (NGF), which leads to the recruitment of the $\mathrm{CAP} / \mathrm{c}-\mathrm{Cbl}$ complex to TrkA. Since the fibroblast growth factor substrate 2 (FRS2) is also capable of binding to TrkA and CAP, it might be possible that the $\mathrm{CAP} / \mathrm{c}-\mathrm{Cbl}$ complex is recruited to TrkA by its interaction with FRS2. Most likely by the scaffolding activity of flotillin-1, the complex including the TrkA receptor is translocated to lipid rafts for a proper initiation and amplification of the downstream signal.

Recently, we have demonstrated that flotillin-1 directly interacts in vitro and in vivo with FRS2, a protein suggested to function in insulin signaling (106), by means of its N-terminal phosphotyrosine binding (PTB) domain and competes for the binding with FGFR (89). Interestingly, FRS2 was shown to interact directly through its PTB domain with TrkA in a phosphotyrosine-dependent manner after NGF stimulation $(107,108)$. Furthermore, we showed that in addition to flotillin-1, FRS2 also binds CAP by means of both its PTB domain 
and the C-terminal domain. In CAP, the SoHo domain in cooperation with the third SH3 domain mediate FRS2 binding (89). Both flotillin-1 and CAP bind to the PTB domain of FRS2, which suggests that CAP and flotillin-1 may compete for FRS2 binding to regulate the formation of signaling complexes.

\section{Animal models for the function of flotillins}

Flotillins are highly conserved proteins which are assumed, mainly on the basis of cell culture studies, to play multifaceted roles in developmental regulation. Hoehne et al. carried out initial studies of loss- and gain-of function of flotillins in wild type and mutant Drosophila melanogaster to investigate their role during morphogenesis and organogenesis (83). According to this study, flotillin proteins are highly expressed in embryonic neuronal tissues, and during later developmental stages flotillins showed an enhanced expression pattern in axon fascicles (83). Additionally, flotillin specific antibodies labeled in particular the Drosophila mushroom body, which is a paired structure of insect central brain responsible for higher-order sensory integration and learning. Flotillins showed a categorical neuronal expression, as antibody staining was restricted to optic lobes and central brain but was not observed in glial cells. A P-element-induced null mutant for flotillin-2 was viable, fertile and with no apparent deficits in behavior or overall activity. In this flotillin-2 null mutant, the expression of flotillin-1 was undetectable even though the mRNA transcript was present (83). Conversely, overexpression of flotillin-1 and flotillin-2 proteins either alone or together in the eye imaginal discs lead to mislocalization of adhesion molecules of the immunoglobulin superfamily (IgCAMs; please also refer to the chapter "Functions of flotillins in cell migration and adhesion"). Additionally, the adult flies showed a variable phenotype affecting bristles, ocelli, eyes, wings, abdominal segments and thoracic structures. A severe wing phenotype, including blistering and melanotic cells, was caused by flotillin overexpression in wing imaginal discs. Moreover, a later study showed that flotillin-2 deficiency resulted in reduced spreading of the morphogens Wnt and hedgehog, whereas flotillin-2 overexpression resulted in increased morphogen secretion and expanded diffusion. These findings qualify flotillin-2 as a novel component of the Wnt and hedgehog secretion pathway in Drosophila (64).

Flotillins are also implicated to regulate axon regeneration in a zebrafish model. As a consequence of gene duplication in fish, there are two copies of each flotillin gene. To understand the essential role of flotillins in axon regeneration, specific morpholino (Mo) antisense nucleotides were used to transiently downregulate flotillin-1a, flotillin-1b and flotillin-2a in zebrafish (60). Interestingly, the number of retinal ganglion cells (RGC) undergoing axon regeneration was reduced by $69 \%$ in flotillin-Mo treated eyes after 7 days (60). Recently, a zebrafish model was employed to study the role of flotillins in cholera intoxication. Consequently, flotillin-1 and flotillin-2 knockdown prevented cholera intoxication in zebrafish embryos. Moreover, cholera toxin subunit B (CTxB) colocalized with flotillins at the plasma membrane and in endosomes in COS1 cells (109). Thus, these results suggest flotillins as essential elements for axon regeneration and indicate a novel cholera intoxication in vivo model dependent on flotillins for trafficking between plasma membrane/endosomes to the ER in the zebrafish. 
Cell migration is an essential mechanism during the development and maintenance of multicellular organisms. It requires the constant turnover of cell-matrix adhesion structures, e.g., integrin-based focal contacts. Results presented by Ludwig et al. (110) strongly suggest a functional role of flotillin microdomains during neutrophil migration, in uropod formation, and in the regulation of myosin IIa activity. They generated and characterized C57BL6/J mice lacking exon 3-8 of the gene for flotillin-1. In line with the observations in Drosophila, these flotillin-1- mice are viable, fertile, and have no readily apparent phenotype. On the cellular level, absence of flotillin-1 resulted in a greatly reduced flotillin-2 expression, and the residual flotillin-2 was not found in detergent-resistant fractions of flot-1 - MEF cells. These data suggest that deletion of flotillin-1 leads to the abrogation of flotillin microdomain function. Furthermore, isolated flotillin-1-- neutrophils exhibited a defect in chemotaxis when exposed to the chemoattractant N-formyl-Met-Leu-Phe (fMLP). Previous reports have already shown that, upon stimulation with chemoattractants, flotillin microdomains rapidly distribute to the uropod, a contractile structure at the back of leukocytes $(32,111)$. This suggested that these microdomains could participate in polarization and/or chemotaxis. Ludwig et al. were also able to show by mass spectrometry of isolated flotillin microdomains and immunoprecipitation of flotillin-2-EGFP an association of flotillin microdomains with myosin IIa, $\alpha$-spectrin and $\beta$-spectrin. Flotillin overexpression led to an increase of myosin IIa activity, whereas the loss of flotillins caused a reduction as measured by using phosphospecific antibodies (110). The regulatory effect of flotillins on myosin IIa activity strengthens the assumption that both proteins exhibit a functional role during cell migration, as myosin IIa was shown to act as a negative regulator of epithelial cell migration, and its ablation results in severe defects in focal adhesion formation and actin stress fiber organization $(112,113)$.

\section{Functional significance of phosphorylation and oligomerization of flotillins}

Recent data from several groups have revealed flotillins as important regulators of vital cellular processes such as signaling and endocytosis. One major challenge in the future will be to dissect how the oligomerization and the covalent modifications of flotillins affect their function during these processes. Endocytosis of flotillins appears to be influenced by upstream signaling $(30,49,56)$ and requires clustering of flotillin-1 and flotillin-2 molecules $(34,56)$. We have shown that EGF stimulation results in uptake of flotillins hetero-oligomers from the plasma membrane, and that hetero-oligomerization appears to be required for the endocytosis $(30,56)$. Mutation of a single Tyr residue, Tyr163, in flotillin-2 is sufficient to prevent the EGF-induced endocytosis of flotillins (30), and this mutation impairs the heterooligomerization of flotillin-2 with flotillin-1 (56). Flotillins are phosphorylated by the Src family kinases cSrc and Fyn $(4,49)$, but the direct role of Tyr phosphorylation in the endocytosis is still under debate. Our data show that inhibition of cSrc kinase, the activity of which is necessary for flotillin-2 phosphorylation (30), does not impair the endocytosis of flotillin-2 (16), whereas the mutation of the single Tyr residue Y163 does (30). Thus, it is more likely that this mutation affects the oligomerization of flotillins and thereby also 
endocytosis. However, phosphorylation of flotillins by the Fyn kinase appears to be sufficient to induce endocytosis of flotillins (49), suggesting that the Src family kinases might play different roles in terms of flotillin function. It is also important to keep in mind that PKC has recently been shown to phosphorylate flotillin-1 in Ser315, and that this phosphorylation is required for the endocytosis of the dopamin transporter DAT (74). Thus, phosphorylation seems to be an important direct or indirect regulator of flotillin trafficking.

Not only phosphorylation but also covalent modifications with fatty acids myristate and palmitate take place in the case of flotillins $(3,4,53)$. Mutation of Gly2 prevents the myristoylation and the subsequent palmitoylation of flotillin-2, thus also inhibiting its membrane association and rendering the protein soluble (4). Recently, the palmitoyl transferase DHHC5 was shown to palmitoylate flotillin-2 (53). Interestingly, growth factor withdrawal, which induces neuronal differentiation, resulted in rapid degradation of DHHC5 (53), implicating that palmitoylation might be important for the well established function of flotillins in neurite outgrowth in cultured neuronal cells. Since the soluble, nonmodified flotillin-2 with Gly2Ala mutation seems to function as a dominant negative protein during cell-matrix adhesion (30), and successive removal of the three palmitoylation sites affect the membrane association of flotillin-2 (4), the fatty acid modifications, especially the reversible palmitoylation, are most likely important for the regulation of flotillin function. Accordingly, we have observed that flotillin-2 expression is necessary for the membrane association of flotillin-1, which is not myristoylated and only palmitoylated in a single residue. Thus, ectopic expression of flotillin-1 in flotillin-2 depleted cells results in a soluble protein that is not capable of exerting its function (our unpublished data).

Our recent findings revealed flotillin-1 as a MAPK regulator (27). However, no data are so far available on the role of flotillin modifications such as phosphorylation in the regulation of MAPK signaling. Intriguingly, another SPFH/PHB family protein, prohibitin-1, has been shown to regulate an earlier step during MAPK signaling, namely signaling by the small GTPase Ras and RAF kinase $(114,115)$. Although the most evident cellular localization of prohibitin is the inner mitochondrial membrane, various studies have now shown that prohibitin is a substrate of several cytoplasmic kinases such as Akt, and it becomes Tyr phosphorylated upon insulin stimulation of cells $(116,117)$. Thus, at least a fraction of prohibitin must be found in another cellular localization such as the plasma membrane. Importantly, prohibitin has been shown to be essential for cell migration that is induced by the Ras-Raf-MAPK pathway (115), and very recent findings show that phosphorylation of prohibitin, which takes place in a raft domain, is necessary for the Ras-dependent enhancement of cellular metastasis (118), verifying the results of Rajalingam et al. (115). Thus, there are several parallels in prohibitin and flotillin function, including MAPK signaling and cell migration, although they appear to be involved in different steps of the signaling. Since some evidence for a hetero-oligomerization of different SPFH-PHB family members, namely prohibitin and stomatin family protein SLP-2 (119) has been presented, it would be important to study if flotillins and prohibitins are capable of hetero-oligomerizing with each other and how this affects their function, especially with respect to the MAPK signaling. In the future, it will also be of interest to study if the phosphorylation- 
incompatible forms of flotillins cause aberrant signaling and if this effect is linked on phosphorylation at specific sites. Our previous data show that the function of flotillin-2 in cell-matrix association is indeed dependent on specific Tyr residues, the mutations of which affect cell spreading (30). Similarly, mutations of phosphorylated residues in flotillin-1 might affect its role in MAPK signaling and its interaction with the MAPK proteins.

\section{Flotillins in human diseases}

\section{Role of flotillins in cancers}

Early findings already indicated that flotillins may be involved in the regulation of the actin cytoskeleton. Overexpression of flotillin-2 was found to result in induction of filopodia-like protrusions and changes in the cytoskeleton $(4,11)$. Furthermore, flotillins have been shown to be important for the polarization of various immune cells such as $\mathrm{T}$ cells and neutrophils $(29,32,33,37,110,111)$, and to be involved in the regulation of the activity of various small GTPases that control e.g. the remodeling of the actin cytoskeleton $(36,57)$. In fact, flotillin-2 has even been suggested to directly interact with actin (36), although this has not been verified in further studies. Thus, there are very strong implications that flotillins are required for a proper regulation of the actin cytoskeleton, which is necessary for e.g. the control of cell migration. Consequently, taking also into account the numerous reports on the involvement of flotillins in signaling, it is evident that flotillins are critical factors that regulate the cell physiology and cell growth. Thus, the findings showing that flotillins are involved in some types of cancer are well in accordance with this function.

The strongest evidence for the role of flotillins in cancer to date comes from studies on human malignant melanoma. The direct association of flotillins with human cancers was shown in 2004 when the group of M. Duvic demonstrated that flotillin-2 is highly expressed in various melanoma cell lines, and that flotillin-2 expression correlated with the progression of human melanoma (120). Furthermore, the thickness of primary melanoma lesions, so-called Breslow depth, which is a prognostic marker for the malignancy of a melanoma lesion, showed a correlation with the increased flotillin-2 expression (121). Importantly, benign, non-malignant cells could be converted into tumorigenic, metastatic ones upon overexpression of flotillin-2 (120). Furthermore, flotillin-2 was shown to be associated with the thrombin receptor PAR1, the expression of which was dependent on the level of flotillin-2 protein (120).

Lin et al. detected a high expression of flotillin-1 in the majority of the breast cancer specimens they studied (66). Furthermore, they revealed a significant correlation between the expression level of flotillin-1 and poor survival of the patients, suggesting that flotillins might prove useful as novel diagnostic markers for cancer. Knockdown of flotillin-1 was shown to result in inhibition of Akt activity and increased transcriptional activity of the transcription factor FOXO3a. Knockdown of flotillin-1 also inhibited the proliferation of breast cancer cell lines by inducing a G1-S phase arrest of the cell cycle. In line with this, the expression of the cyclin dependent kinase (CDK) regulator cyclin D1 was reduced after flotillin-1 silencing, whereas that of the CDK inhibitors $\mathrm{p} 21^{\text {Cip } 1}$ and $\mathrm{p} 27^{\mathrm{Kip} 1}$ was considerably 
increased (66), suggesting that flotillin-1 is an important regulator of the cell cycle. This result is strongly supported by our findings showing that in flotillin-1 knockdown cells, cyclin D1 expression after EGF stimulation is severely impaired (27).

Although flotillins evidently play a role in cancer and their overexpression is associated with malignancy, very little is known about their transcriptional regulation. Flotillin-2 was shown to be a transcriptional target of the p53 family transcription factors p63 and p73 but not of p53 (122). We have recently addressed this question more in detail and shown that flotillins are transcriptionally regulated by the Extracellularly Regulated Kinase signaling and by the Retinoid X Receptor (Banning et al., in revision). Due to their important role in cancer, a deeper knowledge on the transcriptional regulation of flotillins is essential for understanding the mechanisms of malignancy that results from flotillin upregulation.

\section{Implications of flotillins in Alzheimer's Disease and other neurodegenerative disorders}

Alzheimer's disease (AD) is the most common age-related neurodegenerative disorder whose most prominent pathological hallmarks include neuronal loss, neurofibrillary tangle formation and senile plaques throughout the brain cortex. The major component of the senile plaques is the amyloid $\beta$ peptide $(\mathrm{A} \beta)$ comprising of $40-42$ residues, which is generated by a proteolytic cleavage of a large transmembrane precursor protein named amyloid $\beta$ precursor protein (APP). APP is subjected to proteolytic processing at several sites by a group of proteases called secretases. Sequential cleavage of APP by $\beta$-secretase $(\beta-$ site APP cleaving enzyme or BACE) and $\gamma$-secretases generates A $\beta$ and is hence termed the amyloidogenic pathway (reviewed in (123-126)). APP can also be processed through a nonamyloidogenic pathway, in which the $\alpha$-secretase cleaves within the $A \beta$ sequence, thereby precluding the formation of $A \beta$. It has been strongly implicated that the amyloidogenic processing of APP takes place in lipid rafts, which is supported by the findings showing that APP can be detected in rafts isolated from cortical neurons (124). Besides APP, other AD associated raft resident proteins include BACE-1 (127), endoproteolytic fragments of presenilin-1 (PS-1) and the presenilins (128, 129). Compelling evidence from various research groups shows that the flotillin family of proteins is partially associated with AD pathology and trafficking $(47,130,131)$. Flotillins are considered as classic raft markers in neuronal tissue since they show an abundant expression in the brain, typically in pyramidal neurons and astrocytes. Kokubo et al. detected flotillin-1-positive rafts in the plasma membrane and membranes of intracellular organelles in rat brain tissue by using immunoelectron microscopy (47). Consequently, they showed that in APP transgenic mice, flotillin- 1 and $A \beta 42$ colocalized in approximately $10 \%$ of flotillin- 1 positive rafts, and that flotillin-1 expression increased with the accumulation of $A \beta(132)$.

Parallel studies in brain sections from AD, Down syndrome and non-demented subjects with plaques showed enhanced flotillin-1 expression with the progression of AD (130). Further insight was provided by Rajendran et al. who demonstrated that in AD patients, A $\beta$ accumulates and is located in flotillin-1 positive endosomes. They also showed that flotillin1 was not only linked with intracellular $A \beta$ in transgenic mice but was also present in extracellular plaques of $\mathrm{AD}$ patient brain sections (131). Furthermore, comparison of 
flotillin-1 immunoreactivity in the hippocampus, the amygdala and the isocortex of AD and control patients implicated that flotillin-1 principally accumulates in lysosomes of tangle bearing neurons during the advanced stages of $\mathrm{AD}$ (133). To date, the precise functional significance of APP and flotillin association is enigmatic. Schneider et al. proposed that flotillins could function as scaffolding proteins, forming a platform for clustering of APP and consequent endocytosis. Accordingly, flotillin-2 knockdown in mouse neuroblastoma cells impaired APP endocytosis and processing, resulting in reduced A $\beta$ production (75). It has previously been shown that the intracellular domain of APP directly interacts with flotillin-1 (134). Our unpublished findings suggest that both flotillin-1 and flotillin-2 directly interact with APP, suggesting that both flotillins could facilitate recruitment of APP into microdomains.

Flotillins and other lipid raft-associated proteins might also be involved in other human neuronal diseases. The study by Shin et al. (135) showed an increased level of the lipid raft associated proteins caveolin-1, -2 , and -3 in the spinal cord during the early stage of experimental autoimmune encephalomyelitis (EAE). EAE is an experimental animal model, mostly used in rodents, of inflammatory demyelinating diseases of the central nervous system (CNS), including multiple sclerosis (reviewed in (136)). Similar results with the caveolin data were obtained for flotillin-1 during the peak stage of EAE. Immunohistochemical analysis showed an enhanced expression of flotillin- 1 in the dorsal horn lamina and in some flotillin-1-positive macrophages and astrocytes, in which it colocalized with the lysosomal marker cathepsin D (137). Due to that, they postulated that flotillin-1 inherits an important function in immune and neuronal cells within the CNS during EAE, possibly via the activation of signal transduction and lysosomal activity.

Parkinson's disease (PD) is one of the most common, progressive neurological disorders. The pathological hallmark of PD is the extensive loss of dopamine secreting cells within the substantia nigra, particularly affecting the ventral component of the pars compacta (reviewed in (138)), which can lead to uncontrolled muscle contraction and movement, dementia, depression and anxiety. Flotillin-1 staining in rat brain sections showed a prominent labeling in the cytoplasm of catecholamine releasing cells (dopamine, norepinephrine, and epinephrine) and an upregulated gene expression of flotillin-1 in the substantia nigra of Parkinson's brain by RT-PCR (139). Although the molecular function of flotillin-1 in Parkinson's disease is still not known, this study implies that flotillin-1 may be involved in the neuronal changes occurring during Parkinson's disease.

Flotillins and pathogens

During evolution, many pathogens have developed mechanisms to use host-cell lipid rafts as signaling and entry platforms to escape the host immune system (reviewed in $(140,141)$ ). Several studies demonstrated that lipid rafts are often used as entry sites for bacteria, e.g., Salmonella enterica (142) or Listeria monocytogenes (143). In regard to flotillins, Li et al. (144) showed that flotillin-1 accumulates at bacteria entry sites and around intracellular enteropathogenic Escherichia coli (EPEC). This bacterium causes diarrheal disease by disrupting the integrity of tight junctions, which are essential structures for physiological 
homeostasis and defense against pathogen invasion. Furthermore, they detected a shift of flotillin-1 and occludin, a major protein of tight junctions, from Triton X-100 insoluble fractions to Triton X-100 soluble fractions following EPEC infection. This led to the conclusion that the loss of tight junction barrier function might be accounted to the redistribution of occludin and flotillin-1 after EPEC infection.

Not only bacteria use lipid raft domains as preferred entry sites in host-cells, but also various enveloped and non-enveloped viruses are dependent on an intact lipid raft structure and the presence of cholesterol for successful virus entry (reviewed in (145)). In the case of the retrovirus human immunodeficiency virus-1 (HIV-1), flotillin-1 was suggested to play a role of in the cellular response to the viral infection since the protein level of flotillin- 1 was increased in peripheral blood mononuclear cells after treatment with the HIV-1 component gp120 (146). The primary HIV-1 receptor CD4 and the co-receptor CCR5, both of which are important for HIV-1 host-cell entry, were also shown to associate with flotillin-1 containing lipid rafts in monocytes (147). Recently, flotillin-1 was also identified as an interaction partner of the overexpressed rhesus monkey TRIM5 $\alpha$ (148). TRIM5 $\alpha$ is a member of tripartite motif (TRIM) protein family, and plays an important role during host cell defense against retroviruses such as HIV-1 especially in old world monkeys, but not in humans (reviewed in (149)). The function of flotillin-1/TRIM5 $\alpha$ interaction is still unknown, as flotillin-1 knockdown or overexpression did not affect HIV-1 transduction efficiency, leading to the suggestion that flotillin-1 at least plays no role in the post-entry restriction of HIV-1 in fetal rhesus monkey kidney (FRhK4) cells.

\section{Flotillins and diabetes mellitus}

Flotillins seem to be of functional relevance in insulin signaling (see above) and the metabolic disease diabetes mellitus, as first demonstrated by Baumann et al. (63). A year later, James et al. showed an increased protein level of flotillin and caveolin-1 in muscle tissue and myoblast lysates derived from stroke-prone spontaneously hypertensive (SHRSP) rats as compared to male Wistar-Kyoto control rats (150). It is important to note that SHRSP rats are a model of human insulin resistance, defined by the decreased ability of cells or tissues to respond to physiological levels of insulin especially in skeletal muscles and adipose tissue. Insulin resistance is of major importance in several human diseases, e.g. diabetes type 2 (151). The results of both the above studies lead to the assumption that flotillins might play a role in insulin signaling in the skeletal muscle and adipose tissue. Furthermore, during steady state conditions, flotillin-1 was found in perinuclear regions in differentiated skeletal muscle cells, but at the plasma membrane in adipocytes (31). This observation suggested that the role of flotillin-1 in targeting GLUT4 to the plasma membrane after insulin stimulation may be different between muscle and adipose tissue. In fact in skeletal muscle cells, the insulin-stimulated signaling cascade leading to the translocation of GLUT4 to the plasma membrane is dependent on flotillin-1 and the musclespecific protein caveolin-3 (103).

Lipid rafts also play a role in diabetic xerostomia, or dry mouth. This disease, when associated with diabetes mellitus, is caused by degenerative changes in the salivary glands 
leading to increased infectious conditions in the oral cavity and in the long-range aggravates the risk of atherosclerosis and cardiovascular diseases (reviewed in (152)). Wang et al. (153) tried to clarify the mechanisms leading to diabetic xerostomia by testing the subcellular localization of aquaporin 5 (AQP5), a water channel protein, in parotid glands of steptozotocin-induced diabetic rats during stimulation with the muscarinic agonist cevimeline. In parotid acinar cells derived from control rats, AQP5 colocalized with flotillin2 in the cytoplasm and at the apical plasma membrane ten minutes after cemivelime treatment. In contrast, in the parotid acinar cells derived from diabetic rats, the translocation of these proteins did not occur. In summary, the results showed that AQP5 translocation is a lipid raft-dependent process and inhibition of the muscarinic agonist-induced translocation might be the cause of diabetic xerostomia.

\section{Conclusions}

It has become evident that flotillins play a key role in the regulation of cellular signaling and membrane trafficking. Although flotillins are not essential for life in metazoans, including mammals, as shown by the recent studies in flotillin knockout mice, they appear to be extremely important for many processes in cultured cells. This would suggest that especially mammals are capable compensating for the loss of flotillins by upregulating the expression of other proteins and thus overcoming the defects in e.g. signaling and cell adhesion. This feature is observed in both our flotillin-2 knockout mice and cultured cells with a constitutive flotillin knockdown (our unpublished data). In the future, it will be important to study what the molecular mechanisms of the compensation of loss of flotillin function might be. It will also be of major interest to study if the original function of flotillins in neuronal regeneration can also be observed in the mammalian knockout model. Furthermore, the functional significance of flotillin modifications can now be studied in the context of mammalian organisms when knockout/knock-in mice with specific mutations become available. Thus, the recent development of flotillin-1 and flotillin-2 knockout mice has brought the studies of flotillin functions to a new exciting era, and important data on the physiological function of flotillins can be expected in the near future.

\section{Author details}

Nina Kurrle, Bincy John, Melanie Meister and Ritva Tikkanen*

Institute of Biochemistry, Medical Faculty, University of Giessen, Giessen, Germany

\section{Acknowledgement}

The research in the Tikkanen lab is supported by the German Research council DFG (Grants Ti291/6-1 and Ti291/6-2), the Von Behring-Röntgen Foundation and the state of Hessen (LOEWE program "Non-neuronal Cholinergic Systems"), whose support is gratefully acknowledged.

\footnotetext{
${ }^{*}$ Corresponding Author
} 


\section{References}

[1] Bickel PE, Scherer PE, Schnitzer JE, Oh P, Lisanti MP, Lodish HF. Flotillin and epidermal surface antigen define a new family of caveolae-associated integral membrane proteins. J Biol Chem. 1997 May 23;272(21):13793-802.

[2] Schulte T, Paschke KA, Laessing U, Lottspeich F, Stuermer CA. Reggie-1 and reggie-2, two cell surface proteins expressed by retinal ganglion cells during axon regeneration. Development. 1997 Jan;124(2):577-87.

[3] Morrow IC, Rea S, Martin S, Prior IA, Prohaska R, Hancock JF, et al. Flotillin-1/reggie-2 traffics to surface raft domains via a novel golgi-independent pathway. Identification of a novel membrane targeting domain and a role for palmitoylation. J Biol Chem. 2002 Dec 13;277(50):48834-41.

[4] Neumann-Giesen C, Falkenbach B, Beicht P, Claasen S, Luers G, Stuermer CA, et al. Membrane and raft association of reggie-1/flotillin-2: role of myristoylation, palmitoylation and oligomerization and induction of filopodia by overexpression. Biochem J. 2004 Mar 1;378(Pt 2):509-18.

[5] Salzer U, Prohaska R. Stomatin, flotillin-1, and flotillin-2 are major integral proteins of erythrocyte lipid rafts. Blood. 2001 Feb 15;97(4):1141-3.

[6] Simons K, Ikonen E. Functional rafts in cell membranes. Nature. 1997 Jun 5;387(6633):569-72.

[7] Simons K, Gerl MJ. Revitalizing membrane rafts: new tools and insights. Nat Rev Mol Cell Biol. 2010 Oct;11(10):688-99.

[8] Melkonian KA, Ostermeyer AG, Chen JZ, Roth MG, Brown DA. Role of lipid modifications in targeting proteins to detergent-resistant membrane rafts. Many raft proteins are acylated, while few are prenylated. J Biol Chem. 1999 Feb 5;274(6):3910-7.

[9] Fernow I, Icking A, Tikkanen R. Reggie-1 and reggie-2 localize in non-caveolar rafts in epithelial cells: cellular localization is not dependent on the expression of caveolin proteins. Eur J Cell Biol. 2007 Jun;86(6):345-52.

[10] Schroeder WT, Siciliano MJ, Stewart-Galetka SL, Duvic M. The human gene for an epidermal surface antigen (M17S1) is located at 17q11-12. Genomics. 1991 Oct;11(2):4812.

[11] Hazarika P, Dham N, Patel P, Cho M, Weidner D, Goldsmith L, et al. Flotillin 2 is distinct from epidermal surface antigen (ESA) and is associated with filopodia formation. J Cell Biochem. 1999 Oct 1;75(1):147-59.

[12] Deininger SO, Rajendran L, Lottspeich F, Przybylski M, Illges H, Stuermer CA, et al. Identification of teleost Thy-1 and association with the microdomain/lipid raft reggie proteins in regenerating CNS axons. Mol Cell Neurosci. 2003 Apr;22(4):544-54.

[13] Glebov OO, Bright NA, Nichols BJ. Flotillin-1 defines a clathrin-independent endocytic pathway in mammalian cells. Nat Cell Biol. 2006 Jan;8(1):46-54.

[14] Stuermer CA, Lang DM, Kirsch F, Wiechers M, Deininger SO, Plattner H. Glycosylphosphatidyl inositol-anchored proteins and fyn kinase assemble in noncaveolar plasma membrane microdomains defined by reggie-1 and -2. Mol Biol Cell. 2001 Oct;12(10):3031-45. 
[15] Malaga-Trillo E, Solis GP, Schrock Y, Geiss C, Luncz L, Thomanetz V, et al. Regulation of embryonic cell adhesion by the prion protein. PLoS Biol. 2009 Mar 10;7(3):e55.

[16] Babuke T, Tikkanen R. Dissecting the molecular function of reggie/flotillin proteins. Eur J Cell Biol. 2007 Sep;86(9):525-32.

[17] Lopez D, Kolter R. Functional microdomains in bacterial membranes. Genes Dev. 2010 Sep 1;24(17):1893-902.

[18] Haney $\mathrm{CH}$, Long SR. Plant flotillins are required for infection by nitrogen-fixing bacteria. Proc Natl Acad Sci U S A. 2010 Jan 5;107(1):478-83.

[19] Otto GP, Nichols BJ. The roles of flotillin microdomains--endocytosis and beyond. J Cell Sci. 2012 Dec 1;124(Pt 23):3933-40.

[20] Edgar AJ, Polak JM. Flotillin-1: gene structure: cDNA cloning from human lung and the identification of alternative polyadenylation signals. Int J Biochem Cell Biol. 2001 Jan;33(1):53-64.

[21] Browman DT, Hoegg MB, Robbins SM. The SPFH domain-containing proteins: more than lipid raft markers. Trends Cell Biol. 2007 Aug;17(8):394-402.

[22] Tavernarakis N, Driscoll M, Kyrpides NC. The SPFH domain: implicated in regulating targeted protein turnover in stomatins and other membrane-associated proteins. Trends Biochem Sci. 1999 Nov;24(11):425-7.

[23] Solis GP, Hoegg M, Munderloh C, Schrock Y, Malaga-Trillo E, Rivera-Milla E, et al. Reggie/flotillin proteins are organized into stable tetramers in membrane microdomains. Biochem J. 2007 Apr 15;403(2):313-22.

[24] Volonte D, Galbiati F, Li S, Nishiyama K, Okamoto T, Lisanti MP. Flotillins/cavatellins are differentially expressed in cells and tissues and form a hetero-oligomeric complex with caveolins in vivo. Characterization and epitope-mapping of a novel flotillin-1 monoclonal antibody probe. J Biol Chem. 1999 Apr 30;274(18):12702-9.

[25] Yokoyama H, Fujii S, Matsui I. Crystal structure of a core domain of stomatin from Pyrococcus horikoshii Illustrates a novel trimeric and coiled-coil fold. J Mol Biol. 2008 Feb 22;376(3):868-78.

[26] Ha H, Kwak HB, Lee SK, Na DS, Rudd CE, Lee ZH, et al. Membrane rafts play a crucial role in receptor activator of nuclear factor kappaB signaling and osteoclast function. J Biol Chem. 2003 May 16;278(20):18573-80.

[27] Amaddii M, Meister M, Banning A, Tomasovic A, Mooz J, Rajalingam K, et al. Flotillin$1 /$ reggie-2 plays a dual role in the activation of receptor tyrosine kinase/map kinase signaling. J Biol Chem. 2012 Jan 9.

[28] Langhorst MF, Reuter A, Jaeger FA, Wippich FM, Luxenhofer G, Plattner H, et al. Trafficking of the microdomain scaffolding protein reggie-1/flotillin-2. Eur J Cell Biol. 2008 Jan 29.

[29] Langhorst MF, Reuter A, Luxenhofer G, Boneberg EM, Legler DF, Plattner H, et al. Preformed reggie/flotillin caps: stable priming platforms for macrodomain assembly in T cells. FASEB J. 2006 Apr;20(6):711-3.

[30] Neumann-Giesen C, Fernow I, Amaddii M, Tikkanen R. Role of EGF-induced tyrosine phosphorylation of reggie-1/flotillin-2 in cell spreading and signaling to the actin cytoskeleton. J Cell Sci. 2007 Feb 1;120(Pt 3):395-406. 
[31] Liu J, Deyoung SM, Zhang M, Dold LH, Saltiel AR. The stomatin/ prohibitin/ flotillin/ $\mathrm{HflK} / \mathrm{C}$ domain of flotillin-1 contains distinct sequences that direct plasma membrane localization and protein interactions in 3T3-L1 adipocytes. J Biol Chem. 2005 Apr 22;280(16):16125-34.

[32] Rajendran L, Beckmann J, Magenau A, Boneberg EM, Gaus K, Viola A, et al. Flotillins are involved in the polarization of primitive and mature hematopoietic cells. PLoS One. 2009;4(12):e8290.

[33] Rajendran L, Masilamani M, Solomon S, Tikkanen R, Stuermer CA, Plattner H, et al. Asymmetric localization of flotillins/reggies in preassembled platforms confers inherent polarity to hematopoietic cells. Proc Natl Acad Sci U S A. 2003 Jul 8;100(14):8241-6.

[34] Frick M, Bright NA, Riento K, Bray A, Merrified C, Nichols BJ. Coassembly of flotillins induces formation of membrane microdomains, membrane curvature, and vesicle budding. Curr Biol. 2007 Jul 3;17(13):1151-6.

[35] Hansen CG, Nichols BJ. Molecular mechanisms of clathrin-independent endocytosis. J Cell Sci. 2009 Jun 1;122(Pt 11):1713-21.

[36] Langhorst MF, Solis GP, Hannbeck S, Plattner H, Stuermer CA. Linking membrane microdomains to the cytoskeleton: regulation of the lateral mobility of reggie-1/flotillin2 by interaction with actin. FEBS Lett. 2007 Oct 2;581(24):4697-703.

[37] Affentranger S, Martinelli S, Hahn J, Rossy J, Niggli V. Dynamic reorganization of flotillins in chemokine-stimulated human T-lymphocytes. BMC Cell Biol. 2011;12:28.

[38] de Gassart A, Geminard C, Fevrier B, Raposo G, Vidal M. Lipid raft-associated protein sorting in exosomes. Blood. 2003 Dec 15;102(13):4336-44.

[39] Staubach S, Razawi H, Hanisch FG. Proteomics of MUC1-containing lipid rafts from plasma membranes and exosomes of human breast carcinoma cells MCF-7. Proteomics. 2009 May;9(10):2820-35.

[40] Street JM, Barran PE, Mackay CL, Weidt S, Balmforth C, Walsh TS, et al. Identification and proteomic profiling of exosomes in human cerebrospinal fluid. J Transl Med. 2012;10:5.

[41] Strauss K, Goebel C, Runz H, Mobius W, Weiss S, Feussner I, et al. Exosome secretion ameliorates lysosomal storage of cholesterol in Niemann-Pick type C disease. J Biol Chem. 2010 Aug 20;285(34):26279-88.

[42] Dermine JF, Duclos S, Garin J, St-Louis F, Rea S, Parton RG, et al. Flotillin-1-enriched lipid raft domains accumulate on maturing phagosomes. J Biol Chem. 2001 May 25;276(21):18507-12.

[43] Santamaria A, Castellanos E, Gomez V, Benedit P, Renau-Piqueras J, Morote J, et al. PTOV1 enables the nuclear translocation and mitogenic activity of flotillin-1, a major protein of lipid rafts. Mol Cell Biol. 2005 Mar;25(5):1900-11.

[44] Gkantiragas I, Brugger B, Stuven E, Kaloyanova D, Li XY, Lohr K, et al. Sphingomyelinenriched microdomains at the Golgi complex. Mol Biol Cell. 2001 Jun;12(6):1819-33.

[45] Lang DM, Lommel S, Jung M, Ankerhold R, Petrausch B, Laessing U, et al. Identification of reggie-1 and reggie- 2 as plasmamembrane-associated proteins which cocluster with activated GPI-anchored cell adhesion molecules in non-caveolar micropatches in neurons. J Neurobiol. 1998 Dec;37(4):502-23. 
[46] Pust S, Dyve AB, Torgersen ML, van Deurs B, Sandvig K. Interplay between toxin transport and flotillin localization. PLoS One. 2010;5(1):e8844.

[47] Kokubo H, Helms JB, Ohno-Iwashita Y, Shimada Y, Horikoshi Y, Yamaguchi H. Ultrastructural localization of flotillin-1 to cholesterol-rich membrane microdomains, rafts, in rat brain tissue. Brain Res. 2003 Mar 7;965(1-2):83-90.

[48] Carcea I, Ma'ayan A, Mesias R, Sepulveda B, Salton SR, Benson DL. Flotillin-mediated endocytic events dictate cell type-specific responses to semaphorin 3A. J Neurosci. 2010 Nov 10;30(45):15317-29.

[49] Riento K, Frick M, Schafer I, Nichols BJ. Endocytosis of flotillin-1 and flotillin-2 is regulated by Fyn kinase. J Cell Sci. 2009 Apr 1;122(Pt 7):912-8.

[50] Resh MD. Fatty acylation of proteins: new insights into membrane targeting of myristoylated and palmitoylated proteins. Biochim Biophys Acta. 1999 Aug 12;1451(1):1-16.

[51] Johnson DR, Bhatnagar RS, Knoll LJ, Gordon JI. Genetic and biochemical studies of protein N-myristoylation. Annu Rev Biochem. 1994;63:869-914.

[52] Dietrich LE, Ungermann C. On the mechanism of protein palmitoylation. EMBO Rep. 2004 Nov;5(11):1053-7.

[53] Li Y, Martin BR, Cravatt BF, Hofmann SL. DHHC5 protein palmitoylates flotillin-2 and is rapidly degraded on induction of neuronal differentiation in cultured cells. J Biol Chem. 2012 Jan 2;287(1):523-30.

[54] Koegl M, Zlatkine P, Ley SC, Courtneidge SA, Magee AI. Palmitoylation of multiple Src-family kinases at a homologous N-terminal motif. Biochem J. 1994 Nov 1;303 ( Pt 3):749-53.

[55] Ali MH, Imperiali B. Protein oligomerization: how and why. Bioorg Med Chem. 2005 Sep 1;13(17):5013-20.

[56] Babuke T, Ruonala M, Meister M, Amaddii M, Genzler C, Esposito A, et al. Heterooligomerization of reggie-1/flotillin-2 and reggie-2/flotillin-1 is required for their endocytosis. Cell Signal. 2009 Aug;21(8):1287-97.

[57] Langhorst MF, Jaeger FA, Mueller S, Sven Hartmann L, Luxenhofer G, Stuermer CA. Reggies/flotillins regulate cytoskeletal remodeling during neuronal differentiation via CAP/ponsin and Rho GTPases. Eur J Cell Biol. 2008 Dec;87(12):921-31.

[58] Haglund K, Ivankovic-Dikic I, Shimokawa N, Kruh GD, Dikic I. Recruitment of Pyk2 and $\mathrm{Cbl}$ to lipid rafts mediates signals important for actin reorganization in growing neurites. J Cell Sci. 2004 May 15;117(Pt 12):2557-68.

[59] Ludwig A, Otto GP, Riento K, Hams E, Fallon PG, Nichols BJ. Flotillin microdomains interact with the cortical cytoskeleton to control uropod formation and neutrophil recruitment. J Cell Biol. 2011 Nov 15;191(4):771-81.

[60] Munderloh C, Solis GP, Bodrikov V, Jaeger FA, Wiechers M, Malaga-Trillo E, et al. Reggies/flotillins regulate retinal axon regeneration in the zebrafish optic nerve and differentiation of hippocampal and N2a neurons. J Neurosci. 2009 May 20;29(20):660715. 
[61] von Philipsborn AC, Ferrer-Vaquer A, Rivera-Milla E, Stuermer CA, Malaga-Trillo E. Restricted expression of reggie genes and proteins during early zebrafish development. J Comp Neurol. 2005 Feb 14;482(3):257-72.

[62] Stuermer CA. Microdomain-forming proteins and the role of the reggies/flotillins during axon regeneration in zebrafish. Biochim Biophys Acta. 2011 Mar;1812(3):415-22.

[63] Baumann CA, Ribon V, Kanzaki M, Thurmond DC, Mora S, Shigematsu S, et al. CAP defines a second signalling pathway required for insulin-stimulated glucose transport. Nature. 2000 Sep 14;407(6801):202-7.

[64] Katanaev VL, Solis GP, Hausmann G, Buestorf S, Katanayeva N, Schrock Y, et al. Reggie-1/flotillin-2 promotes secretion of the long-range signalling forms of Wingless and Hedgehog in Drosophila. Embo J. 2008 Feb 6;27(3):509-21.

[65] Limpert AS, Karlo JC, Landreth GE. Nerve growth factor stimulates the concentration of TrkA within lipid rafts and extracellular signal-regulated kinase activation through cCbl-associated protein. Mol Cell Biol. 2007 Aug;27(16):5686-98.

[66] Lin C, Wu Z, Lin X, Yu C, Shi T, Zeng Y, et al. Knockdown of FLOT1 impairs cell proliferation and tumorigenicity in breast cancer through upregulation of FOXO3a. Clin Cancer Res. 2011 May 15;17(10):3089-99.

[67] Sugawara Y, Nishii H, Takahashi T, Yamauchi J, Mizuno N, Tago K, et al. The lipid raft proteins flotillins/reggies interact with Galphaq and are involved in Gq-mediated p38 mitogen-activated protein kinase activation through tyrosine kinase. Cell Signal. 2007 Jun;19(6):1301-8.

[68] Helms JB, Zurzolo C. Lipids as targeting signals: lipid rafts and intracellular trafficking. Traffic. 2004 Apr;5(4):247-54.

[69] McMahon HT, Boucrot E. Molecular mechanism and physiological functions of clathrinmediated endocytosis. Nat Rev Mol Cell Biol. 2011 Aug;12(8):517-33.

[70] Staubach S, Hanisch FG. Lipid rafts: signaling and sorting platforms of cells and their roles in cancer. Expert Rev Proteomics. 2011 Apr;8(2):263-77.

[71] Otto GP, Nichols BJ. The roles of flotillin microdomains--endocytosis and beyond. J Cell Sci. 2011 Dec 1;124(Pt 23):3933-40.

[72] Ait-Slimane T, Galmes R, Trugnan G, Maurice M. Basolateral internalization of GPIanchored proteins occurs via a clathrin-independent flotillin-dependent pathway in polarized hepatic cells. Mol Biol Cell. 2009 Sep;20(17):3792-800.

[73] Blanchet MH, Le Good JA, Mesnard D, Oorschot V, Baflast S, Minchiotti G, et al. Cripto recruits Furin and PACE4 and controls Nodal trafficking during proteolytic maturation. EMBO J. 2008 Oct 8;27(19):2580-91.

[74] Cremona ML, Matthies HJ, Pau K, Bowton E, Speed N, Lute BJ, et al. Flotillin-1 is essential for PKC-triggered endocytosis and membrane microdomain localization of DAT. Nat Neurosci. 2011 Apr;14(4):469-77.

[75] Schneider A, Rajendran L, Honsho M, Gralle M, Donnert G, Wouters F, et al. Flotillindependent clustering of the amyloid precursor protein regulates its endocytosis and amyloidogenic processing in neurons. J Neurosci. 2008 Mar 12;28(11):2874-82. 
[76] Ge L, Qi W, Wang LJ, Miao HH, Qu YX, Li BL, et al. Flotillins play an essential role in Niemann-Pick C1-like 1-mediated cholesterol uptake. Proc Natl Acad Sci U S A. 2011 Jan 11;108(2):551-6.

[77] Orth JD, Krueger EW, Weller SG, McNiven MA. A novel endocytic mechanism of epidermal growth factor receptor sequestration and internalization. Cancer Res. 2006 Apr 1;66(7):3603-10.

[78] Sigismund S, Argenzio E, Tosoni D, Cavallaro E, Polo S, Di Fiore PP. Clathrin-mediated internalization is essential for sustained EGFR signaling but dispensable for degradation. Dev Cell. 2008 Aug;15(2):209-19.

[79] Solis GP, Schrock Y, Hulsbusch N, Wiechers M, Plattner H, Stuermer CA. Reggies/Flotillins regulate E-cadherin-mediated cell contact formation by affecting EGFR trafficking. Mol Biol Cell. 2012 Mar 21.

[80] Wiley HS, Cunningham DD. The endocytotic rate constant. A cellular parameter for quantitating receptor-mediated endocytosis. J Biol Chem. 1982 Apr 25;257(8):4222-9.

[81] Masui H, Castro L, Mendelsohn J. Consumption of EGF by A431 cells: evidence for receptor recycling. J Cell Biol. 1993 Jan;120(1):85-93.

[82] Schroeder WT, Stewart-Galetka S, Mandavilli S, Parry DA, Goldsmith L, Duvic M. Cloning and characterization of a novel epidermal cell surface antigen (ESA). J Biol Chem. 1994 Aug 5;269(31):19983-91.

[83] Hoehne M, de Couet HG, Stuermer CA, Fischbach KF. Loss- and gain-of-function analysis of the lipid raft proteins Reggie/Flotillin in Drosophila: they are posttranslationally regulated, and misexpression interferes with wing and eye development. Mol Cell Neurosci. 2005 Nov;30(3):326-38.

[84] Yap AS, Brieher WM, Gumbiner BM. Molecular and functional analysis of cadherinbased adherens junctions. Annu Rev Cell Dev Biol. 1997;13:119-46.

[85] Bodrikov V, Solis GP, Stuermer CA. Prion protein promotes growth cone development through reggie/flotillin-dependent $\mathrm{N}$-cadherin trafficking. J Neurosci. 2011 Dec 7;31(49):18013-25.

[86] Pommereit D, Wouters FS. An NGF-induced Exo70-TC10 complex locally antagonises Cdc42-mediated activation of N-WASP to modulate neurite outgrowth. J Cell Sci. 2007 Aug 1;120(Pt 15):2694-705.

[87] Taulet N, Comunale F, Favard C, Charrasse S, Bodin S, Gauthier-Rouviere C. Ncadherin/p120 catenin association at cell-cell contacts occurs in cholesterol-rich membrane domains and is required for RhoA activation and myogenesis. J Biol Chem. 2009 Aug 21;284(34):23137-45.

[88] Chartier NT, Laine MG, Ducarouge B, Oddou C, Bonaz B, Albiges-Rizo C, et al. Enterocytic differentiation is modulated by lipid rafts-dependent assembly of adherens junctions. Exp Cell Res. 2011 Jun 10;317(10):1422-36.

[89] Tomasovic A, Traub S, Tikkanen R. Molecular Networks in FGF Signaling: Flotillin-1 and Cbl-Associated Protein Compete for the Binding to Fibroblast Growth Factor Receptor Substrate 2. PLoS ONE. 2012;7(1): e29739. doi:10.1371. 
[90] Kato N, Nakanishi M, Hirashima N. Flotillin-1 regulates IgE receptor-mediated signaling in rat basophilic leukemia (RBL-2H3) cells. J Immunol. 2006 Jul 1;177(1):14754 .

[91] Gotoh N, Manova K, Tanaka S, Murohashi M, Hadari Y, Lee A, et al. The docking protein FRS2alpha is an essential component of multiple fibroblast growth factor responses during early mouse development. Mol Cell Biol. 2005 May;25(10):4105-16.

[92] Lax I, Wong A, Lamothe B, Lee A, Frost A, Hawes J, et al. The docking protein FRS2alpha controls a MAP kinase-mediated negative feedback mechanism for signaling by FGF receptors. Mol Cell. 2002 Oct;10(4):709-19.

[93] Wu Y, Chen Z, Ullrich A. EGFR and FGFR signaling through FRS2 is subject to negative feedback control by ERK1/2. Biol Chem. 2003 Aug;384(8):1215-26.

[94] Hofman EG, Ruonala MO, Bader AN, van den Heuvel D, Voortman J, Roovers RC, et al. EGF induces coalescence of different lipid rafts. J Cell Sci. 2008 Aug 1;121(Pt 15):251928.

[95] Ueda Y, Hirai S, Osada S, Suzuki A, Mizuno K, Ohno S. Protein kinase C activates the MEK-ERK pathway in a manner independent of Ras and dependent on Raf. J Biol Chem. 1996 Sep 20;271(38):23512-9.

[96] Zou Y, Komuro I, Yamazaki T, Aikawa R, Kudoh S, Shiojima I, et al. Protein kinase C, but not tyrosine kinases or Ras, plays a critical role in angiotensin II-induced activation of Raf-1 kinase and extracellular signal-regulated protein kinases in cardiac myocytes. J Biol Chem. 1996 Dec 27;271(52):33592-7.

[97] Kouhara H, Hadari YR, Spivak-Kroizman T, Schilling J, Bar-Sagi D, Lax I, et al. A lipidanchored Grb2-binding protein that links FGF-receptor activation to the Ras/MAPK signaling pathway. Cell. 1997 May 30;89(5):693-702.

[98] Roskoski R, Jr. The ErbB/HER receptor protein-tyrosine kinases and cancer. Biochem Biophys Res Commun. 2004 Jun 18;319(1):1-11.

[99] Ribon V, Herrera R, Kay BK, Saltiel AR. A role for CAP, a novel, multifunctional Src homology 3 domain-containing protein in formation of actin stress fibers and focal adhesions. J Biol Chem. 1998 Feb 13;273(7):4073-80.

[100] Ribon V, Printen JA, Hoffman NG, Kay BK, Saltiel AR. A novel, multifuntional c-Cbl binding protein in insulin receptor signaling in 3T3-L1 adipocytes. Mol Cell Biol. 1998 Feb;18(2):872-9.

[101] Kimura A, Baumann CA, Chiang SH, Saltiel AR. The sorbin homology domain: a motif for the targeting of proteins to lipid rafts. Proc Natl Acad Sci U S A. 2001 Jul 31;98(16):9098-103.

[102] Zhang M, Kimura A, Saltiel AR. Cloning and characterization of Cbl-associated protein splicing isoforms. Mol Med. 2003 Jan-Feb;9(1-2):18-25.

[103] Fecchi K, Volonte D, Hezel MP, Schmeck K, Galbiati F. Spatial and temporal regulation of GLUT4 translocation by flotillin-1 and caveolin-3 in skeletal muscle cells. FASEB J. 2006 Apr;20(6):705-7.

[104] Huang EJ, Reichardt LF. Trk receptors: roles in neuronal signal transduction. Annu Rev Biochem. 2003;72:609-42. 
[105] Kaplan DR, Miller FD. Neurotrophin signal transduction in the nervous system. Curr Opin Neurobiol. 2000 Jun;10(3):381-91.

[106] Delahaye L, Rocchi S, Van Obberghen E. Potential involvement of FRS2 in insulin signaling. Endocrinology. 2000 Feb;141(2):621-8.

[107] Meakin SO, MacDonald JI, Gryz EA, Kubu CJ, Verdi JM. The signaling adapter FRS-2 competes with Shc for binding to the nerve growth factor receptor TrkA. A model for discriminating proliferation and differentiation. J Biol Chem. 1999 Apr 2;274(14):986170 .

[108] Ong SH, Hadari YR, Gotoh N, Guy GR, Schlessinger J, Lax I. Stimulation of phosphatidylinositol 3-kinase by fibroblast growth factor receptors is mediated by coordinated recruitment of multiple docking proteins. Proc Natl Acad Sci U S A. 2001 May 22;98(11):6074-9.

[109] Saslowsky DE, Cho JA, Chinnapen H, Massol RH, Chinnapen DJ, Wagner JS, et al. Intoxication of zebrafish and mammalian cells by cholera toxin depends on the flotillin/reggie proteins but not Derlin-1 or -2. J Clin Invest. 2010 Dec;120(12):4399-409.

[110] Ludwig A, Otto GP, Riento K, Hams E, Fallon PG, Nichols BJ. Flotillin microdomains interact with the cortical cytoskeleton to control uropod formation and neutrophil recruitment. J Cell Biol. 2010 Nov 15;191(4):771-81.

[111] Rossy J, Schlicht D, Engelhardt B, Niggli V. Flotillins interact with PSGL-1 in neutrophils and, upon stimulation, rapidly organize into membrane domains subsequently accumulating in the uropod. PLoS One. 2009;4(4):e5403.

[112] Even-Ram S, Doyle AD, Conti MA, Matsumoto K, Adelstein RS, Yamada KM. Myosin IIA regulates cell motility and actomyosin-microtubule crosstalk. Nat Cell Biol. 2007 Mar;9(3):299-309.

[113] Shih W, Yamada S. Myosin IIA dependent retrograde flow drives 3D cell migration. Biophys J. 2010 Apr 21;98(8):L29-31.

[114] Rajalingam K, Rudel T. Ras-Raf signaling needs prohibitin. Cell Cycle. 2005 Nov;4(11):1503-5.

[115] Rajalingam K, Wunder C, Brinkmann V, Churin Y, Hekman M, Sievers C, et al. Prohibitin is required for Ras-induced Raf-MEK-ERK activation and epithelial cell migration. Nat Cell Biol. 2005 Aug;7(8):837-43.

[116] Ande SR, Gu Y, Nyomba BL, Mishra S. Insulin induced phosphorylation of prohibitin at tyrosine 114 recruits Shp1. Biochim Biophys Acta. 2009 Aug;1793(8):1372-8.

[117] Han EK, McGonigal T, Butler C, Giranda VL, Luo Y. Characterization of Akt overexpression in MiaPaCa-2 cells: prohibitin is an Akt substrate both in vitro and in cells. Anticancer Res. 2008 Mar-Apr;28(2A):957-63.

[118] Chiu CF, Ho MY, Peng JM, Hung SW, Lee WH, Liang CM, et al. Raf activation by Ras and promotion of cellular metastasis require phosphorylation of prohibitin in the raft domain of the plasma membrane. Oncogene. 2012 Mar 12.

[119] Da Cruz S, Parone PA, Gonzalo P, Bienvenut WV, Tondera D, Jourdain A, et al. SLP-2 interacts with prohibitins in the mitochondrial inner membrane and contributes to their stability. Biochim Biophys Acta. 2008 May;1783(5):904-11. 
[120] Hazarika P, McCarty MF, Prieto VG, George S, Babu D, Koul D, et al. Up-regulation of Flotillin-2 is associated with melanoma progression and modulates expression of the thrombin receptor protease activated receptor 1. Cancer Res. 2004 Oct 15;64(20):7361-9.

[121] Doherty SD, Prieto VG, George S, Hazarika P, Duvic M. High flotillin-2 expression is associated with lymph node metastasis and Breslow depth in melanoma. Melanoma Res. 2006 Oct;16(5):461-3.

[122] Sasaki Y, Oshima Y, Koyama R, Maruyama R, Akashi H, Mita H, et al. Identification of Flotillin-2, a Major Protein on Lipid Rafts, as a Novel Target of p53 Family Members. Mol Cancer Res. 2008 Feb 22.

[123] Haass C, Lemere CA, Capell A, Citron M, Seubert P, Schenk D, et al. The Swedish mutation causes early-onset Alzheimer's disease by beta-secretase cleavage within the secretory pathway. Nat Med. 1995 Dec;1(12):1291-6.

[124] Kao SC, Krichevsky AM, Kosik KS, Tsai LH. BACE1 suppression by RNA interference in primary cortical neurons. J Biol Chem. 2004 Jan 16;279(3):1942-9.

[125] Small SA, Gandy S. Sorting through the cell biology of Alzheimer's disease: intracellular pathways to pathogenesis. Neuron. 2006 Oct 5;52(1):15-31.

[126] Tikkanen R, Banning A, Meister M. Trafficking and endocytosis of Alzheimer amyloid precursor protein. in: Dowler BC, editor Endocytosis: Structural Components, Functions and Pathways Nova Publishers pp 1-38. 2010.

[127] Riddell DR, Christie G, Hussain I, Dingwall C. Compartmentalization of beta-secretase (Asp2) into low-buoyant density, noncaveolar lipid rafts. Curr Biol. 2001 Aug 21;11(16):1288-93.

[128] Lee SJ, Liyanage U, Bickel PE, Xia W, Lansbury PT, Jr., Kosik KS. A detergent-insoluble membrane compartment contains A beta in vivo. Nat Med. 1998 Jun;4(6):730-4.

[129] Parkin ET, Hussain I, Karran EH, Turner AJ, Hooper NM. Characterization of detergent-insoluble complexes containing the familial Alzheimer's disease-associated presenilins. J Neurochem. 1999 Apr;72(4):1534-43.

[130] Kokubo H, Lemere CA, Yamaguchi H. Localization of flotillins in human brain and their accumulation with the progression of Alzheimer's disease pathology. Neurosci Lett. 2000 Aug 25;290(2):93-6.

[131] Rajendran L, Knobloch M, Geiger KD, Dienel S, Nitsch R, Simons K, et al. Increased Abeta production leads to intracellular accumulation of Abeta in flotillin-1-positive endosomes. Neurodegener Dis. 2007;4(2-3):164-70.

[132] Kokubo H, Saido TC, Iwata N, Helms JB, Shinohara R, Yamaguchi H. Part of membrane-bound Abeta exists in rafts within senile plaques in Tg2576 mouse brain. Neurobiol Aging. 2005 Apr;26(4):409-18.

[133] Girardot N, Allinquant B, Langui D, Laquerriere A, Dubois B, Hauw JJ, et al. Accumulation of flotillin-1 in tangle-bearing neurones of Alzheimer's disease. Neuropathol Appl Neurobiol. 2003 Oct;29(5):451-61.

[134] Chen TY, Liu PH, Ruan CT, Chiu L, Kung FL. The intracellular domain of amyloid precursor protein interacts with flotillin-1, a lipid raft protein. Biochem Biophys Res Commun. 2006 Mar 31;342(1):266-72. 
[135] Shin T, Kim H, Jin JK, Moon C, Ahn M, Tanuma N, et al. Expression of caveolin-1, -2, and -3 in the spinal cords of Lewis rats with experimental autoimmune encephalomyelitis. J Neuroimmunol. 2005 Aug;165(1-2):11-20.

[136] Constantinescu CS, Farooqi N, O'Brien K, Gran B. Experimental autoimmune encephalomyelitis (EAE) as a model for multiple sclerosis (MS). Br J Pharmacol. 2011 Oct;164(4):1079-106.

[137] Kim H, Ahn M, Moon C, Matsumoto Y, Sung Koh C, Shin T. Immunohistochemical study of flotillin-1 in the spinal cord of Lewis rats with experimental autoimmune encephalomyelitis. Brain Res. 2006 Oct 9;1114(1):204-11.

[138] Davie CA. A review of Parkinson's disease. Br Med Bull. 2008;86:109-27.

[139] Jacobowitz DM, Kallarakal AT. Flotillin-1 in the substantia nigra of the Parkinson brain and a predominant localization in catecholaminergic nerves in the rat brain. Neurotox Res. 2004;6(4):245-57.

[140] Manes S, del Real G, Martinez AC. Pathogens: raft hijackers. Nat Rev Immunol. 2003 Jul;3(7):557-68.

[141] Vieira FS, Correa G, Einicker-Lamas M, Coutinho-Silva R. Host-cell lipid rafts: a safe door for micro-organisms? Biol Cell. 2010 Jul;102(7):391-407.

[142] Knodler LA, Vallance BA, Hensel M, Jackel D, Finlay BB, Steele-Mortimer O. Salmonella type III effectors PipB and PipB2 are targeted to detergent-resistant microdomains on internal host cell membranes. Mol Microbiol. 2003 Aug;49(3):685-704.

[143] Seveau S, Bierne H, Giroux S, Prevost MC, Cossart P. Role of lipid rafts in E-cadherin-and HGF-R/Met--mediated entry of Listeria monocytogenes into host cells. J Cell Biol. 2004 Aug 30;166(5):743-53.

[144] Li Q, Zhang Q, Wang C, Li N, Li J. Invasion of enteropathogenic Escherichia coli into host cells through epithelial tight junctions. FEBS J. 2008 Dec;275(23):6022-32.

[145] Chazal N, Gerlier D. Virus entry, assembly, budding, and membrane rafts. Microbiol Mol Biol Rev. 2003 Jun;67(2):226-37, table of contents.

[146] Cicala C, Arthos J, Selig SM, Dennis G, Jr., Hosack DA, Van Ryk D, et al. HIV envelope induces a cascade of cell signals in non-proliferating target cells that favor virus replication. Proc Natl Acad Sci U S A. 2002 Jul 9;99(14):9380-5.

[147] Carter GC, Bernstone L, Sangani D, Bee JW, Harder T, James W. HIV entry in macrophages is dependent on intact lipid rafts. Virology. 2009 Mar 30;386(1):192-202.

[148] Ohmine S, Sakuma R, Sakuma T, Thatava T, Solis GP, Ikeda Y. Cytoplasmic body component TRIM5\{alpha\} requires lipid-enriched microdomains for efficient HIV-1 restriction. J Biol Chem. 2010 Nov 5;285(45):34508-17.

[149] Nakayama EE, Shioda T. Role of Human TRIM5alpha in Intrinsic Immunity. Front Microbiol. 2012;3:97.

[150] James DJ, Cairns F, Salt IP, Murphy GJ, Dominiczak AF, Connell JM, et al. Skeletal muscle of stroke-prone spontaneously hypertensive rats exhibits reduced insulinstimulated glucose transport and elevated levels of caveolin and flotillin. Diabetes. 2001 Sep;50(9):2148-56. 
[151] Collison M, Glazier AM, Graham D, Morton JJ, Dominiczak MH, Aitman TJ, et al. Cd36 and molecular mechanisms of insulin resistance in the stroke-prone spontaneously hypertensive rat. Diabetes. 2000 Dec;49(12):2222-6.

[152] Ship JA. Diabetes and oral health: an overview. J Am Dent Assoc. 2003 Oct;134 Spec No:4S-10S.

[153] Wang D, Yuan Z, Inoue N, Cho G, Shono M, Ishikawa Y. Abnormal subcellular localization of AQP5 and downregulated AQP5 protein in parotid glands of streptozotocin-induced diabetic rats. Biochim Biophys Acta. 2011 May;1810(5):543-54. 